Sharif University of Technology
Scientia Iranica
Transactions A: Civil Engineering
SCIENTIA
IRAN I CA

\title{
Body waves propagation in a fluid-saturated transversely isotropic poroelastic solid with a potential method
}

\author{
M. Mahmoodian ${ }^{a}$, M. Eskandari-Ghadi ${ }^{b, *}$, and A. Nikkhoo ${ }^{a}$ \\ a. Department of Civil Engineering, Faculty of Engineering, University of Science and Culture, Tehran, P.O. Box 13145-871, Iran. \\ b. School of Civil Engineering, College of Engineering, University of Tehran, Tehran, P.O. Box 11165-4563, Iran.
}

Received 10 October 2019; received in revised form 9 January 2020; accepted 20 April 2020

\author{
KEYWORDS \\ Wave propagation; \\ $P$-wave; \\ $S V$-wave; \\ $S H$-wave; \\ Transversely isotropic; \\ Saturated poroelastic; \\ Simplified $\mathbf{u}-p$ \\ formulation; \\ Scalar potential \\ function.
}

\begin{abstract}
This study is focused on the propagation of plane harmonic body waves in a transversely isotropic linear poroelastic fluid-saturated medium in the framework of the simplified $\mathbf{u}-p$ formulation. A set of two scalar potential functions is employed to decouple the coupled fluid continuity equation and equations of motion, such a way that the governing equations for the potential functions are resulted in the form of either scaled wave motion or a combination of repeated wave motion and transport equation. The velocities and corresponding attenuation coefficients of both longitudinal and transverse waves are extracted from the acoustic equations for the body waves. To show the validity of the analytical solution developed in this paper, the degeneration to the case of a singlephase transversely isotropic, and consequently isotropic solid is presented. The motivation behind this was to provide the possibility of comparing the results of this study and the developed solution with those reported in the literature. Besides, the effects of mechanical and hydraulic parameters of materials on the velocity of propagation and attenuation coefficient of the waves are investigated in more detail. To this end, various synthetic poroelastic transversely isotropic materials are defined, and the dependency of wave motion to these parameters is illustrated by plotting the graphs.

(C) 2021 Sharif University of Technology. All rights reserved.
\end{abstract}

\section{Introduction}

The problem of wave propagation in a fluid-saturated porous medium, such as saturated soils or saturated rocks, is a very important topic in many branches of engineering, e.g., seismology, geophysics, petroleum industry, soil mechanics, pollution, biomechanics, etc. $[1,2]$. Moreover, the phenomenon of fluid flowing

*. Corresponding author. Fax: +982182473660 E-mail addresses: m.mahmoodian@usc.ac.ir (M. Mahmoodian); ghadi@ut.ac.ir (M. Eskandari-Ghadi); nikkhoo@usc.ac.ir (A.Nikkhoo)

doi: $10.24200 /$ sci.2020.54428.3748 through a medium with complex structures plays important roles in the industry, natural environment, and human life [3]. Moreover, due to the interaction between the solid skeleton and the pore fluid, the macro mechanical properties of the two-phase materials are very important, especially in dynamic analysis. Therefore, this topic received a great deal of attention by engineers and since a long time, to explore and understand the physical behavior of a two- or morephase material had been the subject of many research efforts made in this area [4]. On the other hand, the fluid-saturated porous media is different from singlephase media and its mathematical study is much more complicated compared to latter, thus mathematicians showed interest to proceed to it. By chronological 
investigation of multiphase continuum mechanics, we could identify two poroelastic theories including; the Biot's theory [5-9] and the theory of porous media. Today these new emerged theories have an special statute and application. More details of the chronological development of porous media theories could be found in the work of De Boer [10]. The research in the field of poroelastodynamics was initiated by Biot whose papers published in $1956[8,9]$. The topics discussed in these papers were the low and high frequency range, the boundary of which depends on both the viscosity of the fluid and the size of pores [8]. According to this theory, the material parameters have an acceptable physical meaning which could be determined in the laboratory $[11,12]$. This is considered as one of the most important features of Biot's theory.

Generally, the vector of the solid displacement, the vector of seepage velocity, and the pore pressure are used to derive the governing equations in different theories relating to poroelasticity. In a slightly different form, namely $\mathbf{u}-p$ formulation, the seepage velocity is replaced by fluid pressure by virtue of generalized Darcy's law if either Fourier or Laplace transform is also applied. It has been concluded that in geomechanical applications and soil mechanics with mostly lowfrequency acceleration, the complete Biot's theory does not significantly differ from $\mathbf{u}-p$ formulation. Bonnet [13] showed that this choice is sufficient, where the solid displacement, $\mathbf{u}$, and the pore fluid pressure, $p$, are the primary variables. Nevertheless, this formulation exists only in the Laplace or Fourier domain. Hence, a simplified poroelastic model has been introduced to be applied in poroelastic wave propagation to derive and solve the governing differential equations directly in the time domain [14].

Based on the Biot's equations, a simplified formulation for poroelastodynamic problems in lowfrequency has been proposed by Zienkiewicz et al. [15], where the inertial effect due to the relative acceleration between pore fluid and solid phases is ignored. The results of the investigations indicated that the problems with low- frequency accelerations can be treated well by simplified $\mathbf{u}-p$ formulation, with applications in earthquake engineering as an example, while it may be inaccurate for high-frequencies [15-17]. Schanz and Struckmeier [16] showed that the solution from the complete $\mathbf{u}-p$ formulation and the formulation in which the time-derivative of the seepage velocity is omitted (simplified $\mathbf{u}-p$ formulation), are quite similar. Since simplified $\mathbf{u}-p$ formulation contains fewer unknown variables compared to the fully vectored formulations, and since the pore pressure can be obtained directly from this formulation without iterations; a higher computational efficiency could be attained, thus it is convenient to be used to describe the dynamic problems of the saturated porous media [18].
A few studies have been done on elastic wave propagation and its attenuation in poroelastic media. Greetsma and Smit [19] investigated some aspects of wave propagation in poroelastic rock, while Deresiewicz [20,21] and Deresiewicz and Rice [22], using Biot's model, were able to study the effects of boundaries on harmonic wave propagation in a saturated porous solid. Also, Beskos et al. [23] have analyticallynumerically studied the propagation of plane harmonic body waves in an unbounded, fully saturated, elastic rock media characterized by two degrees of porosity. By conducting this study the differences of velocity and attenuation of the double and single porosity models for a certain range of frequency were revealed. Besides, Beskos et al. [24] analytically proved that the propagation of plane harmonic Rayleigh waves in a fully saturated elastic rocky half-space is characterized by two degrees of porosity.

On the other hand, many kinds of rocks located near the earth surface show well-defined fabric elements in the form of layering, bedding, foliation, stratification, jointing, or fissuring. These rocks have anisotropic properties in terms of physical, mechanical, thermal and hydraulic features [25]. Practically, these rocks are often considered as either orthotropic or transversely isotropic materials. Thus, studying the behavior of both orthotropic and transversely isotropic materials is necessary. Regarding the wave propagation in saturated material of this kind some researches are conducted some of which are indicated in the following. Schmitt [26] gave out the dispersion and attenuation curves of plane waves in a transversely isotropic fluidsaturated porous medium. Sharma and Gogna [27] investigated the possibility of the presence of body waves with a real velocity of propagation and Rayleigh waves at the free surface of transversely isotropic poroelastic solids. Carcione [28] gave the fundamentals of wave propagation in anelastic, anisotropic, and porous media, including electromagnetic waves. Also, the effect of anisotropy of a solid skeleton on the propagation of plane waves in the porous medium has been discussed in the work of Carcione [29]. Furthermore, Ba and his co-workers computed the dynamic response of a multilayered transversely isotropic saturated half-space subjected to pore fluid pressure and time-harmonic loads using the exact dynamic stiffness matrix method [30]. In this study, it was found that the dynamic responses between the isotropic and transversely isotropic media can be significantly different, and the transversely isotropic parameters have a significant influence on the solutions. More recently, Zhang and Pan presented a new semi-analytical method for solving the dynamic response of a multilayered transversely isotropic and poroelastic half-space subjected to time-harmonic horizontal and vertical loads buried in the layered halfspace [31]. 
The potential method is an elegant and common method for decoupling a set of coupled linear partial differential equations (PDEs), where the physical aspects of original PDEs may be recognized from the governing equations of the potential functions. One of the famous sets of potential functions for one-phase isotropic material is the combination of a divergence-free and a curl-free vector function, known as Helmholtz decomposition [32]. Lu and Jeng [33] used the same decomposition, with some modifications to uncouple the governing wave and transport equations of Biot's theory. Also, an alternative solution method to the Helmholtz decomposition was developed by Pooladi and his co-authors [34] that could be utilized to solve the wave propagation problems in isotropic fluid-saturated poroelastic media. On the other hand, some remarkable past potential functions for a transversely isotropic medium are presented by Lekhnitskii-Hu-Nowacki [35] for elastostatic and by Eskandari-Ghadi [36] for elastodynamic boundary value problems. A complete solution for the general elastodynamics boundary value problem in a linear elastic cross-anisotropic mono-axial-convex domain in terms of two scalar potential functions was introduced by Eskandar-Ghadi [36]. In this solution one of the potential functions is related to $S H$-wave and other to $S V$ - and $P$-waves. Raoofian-Naeeni and EskandariGhadi [37] determined the surface and body wave velocities in terms of both the direction of propagation and elasticity coefficients in a single-phase transversely isotropic media with a depth-wise axis of material symmetry using the above-mentioned scalar potential functions. In this study, the Rayleigh, longitudinal and transverse wave velocities were determined in explicit forms. Furthermore, Pooladi et al. [38] have obtained a pair of scalar potentials to uncouple Biot's equations of motion in transversely isotropic fluidsaturated poroelastic media. It is worth noting that they considered the governing equations in the form of the $\mathbf{u}-p$ formulation. An analytical solution has been presented for stress wave propagation in a saturated porous transversely isotropic half-space under surface traction by Sahebkar and Eskandari-Ghadi [17]. In this study, for the first time, the simplified $\mathbf{u}$ - $p$ formulation was considered, and two scalar potential functions were proposed to decouple the governing PDEs. Moreover, with the help of these potential functions, analytical solutions of many poroelastodynamic problems in the transversely isotropic fluid-saturated media have been studied; some of studies conducted in this area include: [39-42]. Very recently, using the potential functions, Liang et al. investigated the three-dimensional (3D) dynamic responses of a transversely isotropic saturated half-space subjected to uniformly distributed buried loads acting on a circular disk [43].

In the present paper, the propagation of plane harmonic body waves in a transversely isotropic fully saturated medium is studied analytically based on simplified $\mathbf{u}-p$ formulation. In accordance to the assumption that both fluid and solid are transversely isotropic, both hydraulic and mechanical axes of the symmetry of the material are conceived to be depth-wise. With the aid of two scalar potential functions, determined by Sahebkar and Eskandari-Ghadi [17], the coupled fluid continuity equation and equations of motion are decoupled. The longitudinal and transverse velocities and related attenuation coefficients are extracted from the presented body wave equations. The obtained results imply the existence of two compressional and two shear waves. The findings of the present study showed that the $S H$-wave, which represents one of these shear waves, showed the same behavior as in the corresponding single-phase transversely isotropic elastic medium. Also, the expression of velocity and corresponding attenuation coefficient of body waves are given in explicit form for some special direction of propagation and saturated isotropic materials. Besides, under the assumption of incompressible constituents, for some special cases, the slowness are obtained. To prove the validity of the analytical solution given in this study, the results are reduced to the case of a single-phase of transversely isotropic and isotropic itself, which is in agreement with the solutions presented in the literature. Also, to study the effects of hydraulic and mechanical parameters on the behavior of wave motion, various numerical results are plotted for different synthetic poroelastic transversely isotropic materials.

\section{Problem statement and potential functions}

A fluid-filled elastic skeleton with interconnected pores, which itself is transversely isotropic in terms of both fluid and solid with a depth-wise axis of material symmetry is considered. A Cartesian coordinate system with a vertical downward $x_{3}$-axis is attached to the domain, so that both hydraulic and mechanical axes of material symmetry are parallel to the $x_{3}$-axis. Based on Biot's theory, in this paper the simplified $\mathbf{u}-p$ formulation [15] for fully saturated material, in which the relative pore fluid acceleration is neglected, is considered. The equations of motion, in the absence of body force, and the transport equation may be written as [4]:

$$
\begin{aligned}
& \sigma_{i j, j}-\rho \frac{\partial^{2} u_{i}}{\partial t^{2}}=0 \\
& \frac{\partial \zeta}{\partial t}+q_{i, i}=0
\end{aligned}
$$

where $\sigma_{i j}=\sigma_{i j}^{s}-n p \delta_{i j}$ is the total stress defined on the surface of a bulk volume saturated poroelastic, with 


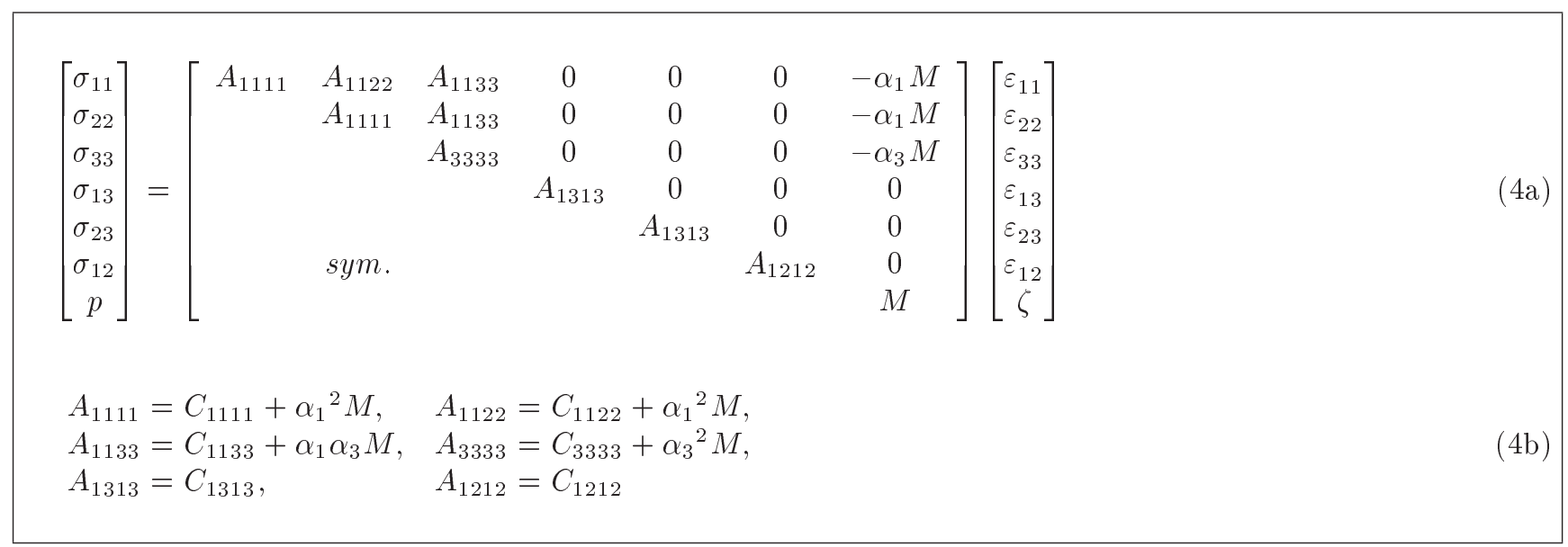

$\sigma_{i j}^{s}$ and $n p \delta_{i j}$ respectively being the stress tensor of the solid and the fluid, where $p$ denotes the pore pressure, as an independent variable and $\delta_{i j}$ is the Kronecker delta. The sign conventions for stress follow that of elasticity, namely, tensile stress is denoted positive. Note that the derivative with respect to the spatial variable $x_{i}$ in Eqs. (1) and (2) is abbreviated by ()$_{, i}$. Besides, the bulk density $\rho=(1-n) \rho_{s}+n \rho_{f}$ with $\rho_{s}$ being the density of the solid and $\rho_{f}$ the density of fluid is used. The sealed pores are considered as a part of the solid, and the porosity, $n$, is defined as the ratio of $n=$ $V_{f} / V$, where $V_{f}$ is the volume of the interconnected pores contained in a sample of bulk volume $V$. Full saturation is expected, leading to $V=V_{f}+V_{s}$ with $V_{s}$ being the volume of the solid. Furthermore, the index $f$ and $s$ denote to the interstitial fluid and the solid, respectively. In addition, $q_{i}=\partial w_{i} / \partial t$ refers to the specific flux of the fluid passing through the surface of a bulk volume. The relative fluid to solid displacement is denoted by $w_{i}=n\left(U_{i}-u_{i}\right)$, where the corresponding displacement of the fluid and the displacement in the solid are $U_{i}$ and $u_{i}$, respectively. Eventually, $\zeta$ is the variation of fluid volume per unit reference volume. Eq. (2) identifies $\zeta$ to describe the motion of the fluid relative to the solid. The dynamic version of Darcy's law, which shows the fluid transport in the interstitial space expressed by the specific flux, by eliminating the second time derivative of the seepage displacement is written in the form of [4]:

$$
q_{i}=-\frac{k_{j}}{\eta}\left(p_{, i}+\rho_{f} \frac{\partial^{2} u_{i}}{\partial t^{2}}\right),
$$

where $k_{j}$ with $j=1$ and 3 are the intrinsic permeability of porous media in any direction in the isotropic plane and in a direction parallel to the material axis of symmetry, respectively, and $\eta$ is introduced as fluid dynamic viscosity. The factor $k_{j} / \eta(j=1$ and 3$)$ in Eq. (3) is usually indicated by $\kappa$ which is called permeability. Moreover, when the frequency of excitation $f_{t}$ exceeds $\pi v / 4 d^{2}$ Darcy's law, which governs the flow in pores, it is not valid [8], where $\nu$ is kinematic viscosity and $d$ is the order of the diameter of the pores. According to this limitation, the relative motion of the fluid in the pores is assumed to be Poiseuille type [8]. In case where the water temperature is $15^{\circ} \mathrm{C}$, Biot [8] found $f_{t}=100 \mathrm{~Hz}$ for $d=10^{-2} \mathrm{~cm}$ and $f_{t}=10^{4} \mathrm{~Hz}$ for $d=10^{-3} \mathrm{~cm}$.

Taking the linear strain-displacement relationships into account for the solid part, $\varepsilon_{i j}=1 / 2\left(u_{i, j}+\right.$ $\left.u_{j, i}\right)$, the constitutive equations in a transversely isotropic saturated porous medium in the Cartesian coordinate system may be written by Eqs. (4a) and (4b) [44] as shown in Box I, where $C_{i j k l}$ are the elasticity coefficients with the property of $C_{1122}=$ $C_{1111}-2 C_{1212}$. The following inequalities should be held to have a positive definite strain energy function $[36,44]$ :

$$
\begin{aligned}
& C_{1111}>\left|C_{1122}\right|, \quad C_{1313}>0, \\
& C_{1111} C_{3333}-2 C^{2}{ }_{1133}+C_{3333} C_{1212}>0, \quad M \geq 0,
\end{aligned}
$$

$\alpha_{1}$ and $\alpha_{3}$ are the Biot's effective stress coefficient in isotropic planes and vertical planes respectively, which are defined as follows [29]:

$$
\begin{aligned}
& \alpha_{1}=1-\frac{C_{1111}+C_{1122}+C_{1133}}{3 K_{s}}, \\
& \alpha_{3}=1-\frac{2 C_{1133}+C_{3333}}{3 K_{s}} .
\end{aligned}
$$

It was shown in [45] that the values of $\alpha_{j}(j=1,3)$ lie within the range of $n \leq \alpha_{j} \leq 1$. In addition, $M$ is the Biot's modulus which can be obtained through [29]:

$$
\frac{1}{M}=\frac{1}{K_{s}}\left(\frac{2 \alpha_{1}+\alpha_{3}}{3}-n\right)+\frac{n}{K_{f}},
$$

with $K_{s}$ and $K_{f}$ being the compression modulus of 
the solid grain and fluid, respectively. With the use of Eqs. (1) through (4), a set of coupled PDEs for the unknowns solid displacement $u_{i}$ and pore pressure $p$, in the Cartesian coordinate system is found as:

$$
\begin{aligned}
& C_{1111} \frac{\partial^{2} u_{1}}{\partial x_{1}^{2}}+\left(\frac{C_{1111}-C_{1122}}{2}\right) \frac{\partial^{2} u_{1}}{\partial x_{2}^{2}}+C_{1313} \frac{\partial^{2} u_{1}}{\partial x_{3}^{2}} \\
& +\left(\frac{C_{1111}+C_{1122}}{2}\right) \frac{\partial^{2} u_{2}}{\partial x_{1} \partial x_{2}}+\left(C_{1133}+C_{1313}\right) \\
& \frac{\partial^{2} u_{3}}{\partial x_{1} \partial x_{3}}-\alpha_{1} \frac{\partial p}{\partial x_{1}}-\rho \frac{\partial^{2} u_{1}}{\partial t^{2}}=0 \\
& \left(\frac{C_{1111}-C_{1122}}{2}\right) \frac{\partial^{2} u_{2}}{\partial x_{1}^{2}}+C_{1111} \frac{\partial^{2} u_{2}}{\partial x_{2}^{2}}+C_{1313} \frac{\partial^{2} u_{2}}{\partial x_{3}^{2}} \\
& +\left(\frac{C_{1111}+C_{1122}}{2}\right) \frac{\partial^{2} u_{1}}{\partial x_{1} \partial x_{2}}+\left(C_{1133}+C_{1313}\right) \\
& \frac{\partial^{2} u_{3}}{\partial x_{2} \partial x_{3}}-\alpha_{1} \frac{\partial p}{\partial x_{2}}-\rho \frac{\partial^{2} u_{2}}{\partial t^{2}}=0, \\
& C_{1313} \frac{\partial^{2} u_{3}}{\partial x_{1}^{2}}+C_{1313} \frac{\partial^{2} u_{3}}{\partial x_{2}^{2}}+C_{3333} \frac{\partial^{2} u_{3}}{\partial x_{3}^{2}} \\
& +\left(C_{1133}+C_{1313}\right) \frac{\partial^{2} u_{1}}{\partial x_{1} \partial x_{3}}+\left(C_{1133}+C_{1313}\right) \\
& \frac{\partial^{2} u_{2}}{\partial x_{2} \partial x_{3}}-\alpha_{3} \frac{\partial p}{\partial x_{3}}-\rho \frac{\partial^{2} u_{3}}{\partial t^{2}}=0, \\
& k_{1} \rho_{f} \frac{\partial^{3} u_{1}}{\partial x_{1} \partial t^{2}}-\alpha_{1} \eta \frac{\partial^{2} u_{1}}{\partial x_{1} \partial t}+k_{1} \rho_{f} \frac{\partial^{3} u_{2}}{\partial x_{2} \partial t^{2}} \\
& -\alpha_{1} \eta \frac{\partial^{2} u_{2}}{\partial x_{2} \partial t}+k_{3} \rho_{f} \frac{\partial^{3} u_{3}}{\partial x_{3} \partial t^{2}}-\alpha_{3} \eta \frac{\partial^{2} u_{3}}{\partial x_{3} \partial t} \\
& +k_{1} \nabla_{12}^{2} p+k_{3} \frac{\partial^{2} p}{\partial x_{3}^{2}}-\frac{\eta}{M} \frac{\partial p}{\partial t}=0,
\end{aligned}
$$

in which $u_{1}, u_{2}$ and $u_{3}$ are the displacement of the solid in $x_{1}-, x_{2}-$ and $x_{3}-$ direction, respectively. The general solution of these equations in a $x_{3}-$ convex medium may be obtained by expressing the displacement $\mathbf{u}=\left(u_{1}, u_{2}, u_{3}\right)$ and the pore pressure $p$ in terms of two scalar potential functions $F$ and $\chi$ as [17];

$$
\begin{aligned}
u_{1}= & -\bar{k}_{1} \beta_{3} \frac{\partial^{2}}{\partial x_{1} \partial x_{3}}\left[\square_{p}^{2}+\frac{\bar{\alpha}_{1} \bar{k}_{3}}{\beta_{3} \bar{k}_{1}}\left(\rho_{f} \frac{\partial^{2}}{\partial t^{2}}-\frac{\eta \bar{\alpha}_{3}}{\bar{k}_{3}} \frac{\partial}{\partial t}\right)\right] F \\
& -\frac{\partial \chi}{\partial x_{2}}, \\
u_{2}= & -\bar{k}_{1} \beta_{3} \frac{\partial^{2}}{\partial x_{2} \partial x_{3}}\left[\square_{p}^{2}+\frac{\bar{\alpha}_{1} \bar{k}_{3}}{\beta_{3} \bar{k}_{1}}\left(\rho_{f} \frac{\partial^{2}}{\partial t^{2}}-\frac{\eta \bar{\alpha}_{3}}{\bar{k}_{3}} \frac{\partial}{\partial t}\right)\right] F \\
& +\frac{\partial \chi}{\partial x_{1}}
\end{aligned}
$$

$$
\begin{aligned}
u_{3}= & \bar{k}_{1}\left[\left(\square_{0}^{2}+\beta_{1} \nabla_{12}^{2}\right) \square_{p}^{2}+\bar{\alpha}_{1} \nabla_{12}^{2}\left(\rho_{f} \frac{\partial^{2}}{\partial t^{2}}\right.\right. \\
& \left.\left.-\frac{\eta \bar{\alpha}_{1}}{\bar{k}_{1}} \frac{\partial}{\partial t}\right)\right] F \\
p=- & \frac{\partial^{2}}{\partial t \partial x_{3}}\left\{-\eta \bar{\alpha}_{3}\left[\square_{0}^{2}+\left(\beta_{1}-\beta_{3} \frac{\bar{\alpha}_{1}}{\bar{\alpha}_{3}}\right) \nabla_{12}^{2}\right]\right. \\
& \left.+\bar{k}_{3} \rho_{f} \frac{\partial}{\partial t}\left[\square_{0}^{2}+\left(\beta_{1}-\beta_{3} \frac{\bar{k}_{1}}{\bar{k}_{3}}\right) \nabla_{12}^{2}\right]\right\} F
\end{aligned}
$$

where:

$$
\begin{aligned}
& \beta_{1}=\frac{C_{1122}+C_{1212}}{C_{1212}}, \quad \beta_{2}=\frac{C_{1313}}{C_{1212}}, \\
& \beta_{3}=\frac{C_{1133}+C_{1313}}{C_{1212}}, \quad \beta_{4}=\frac{C_{3333}}{C_{1212}}, \\
& \beta_{5}=\frac{\eta}{M C_{1212}}, \\
& \bar{k}_{1}=\frac{k_{1}}{C_{1212}}, \quad \bar{k}_{3}=\frac{k_{3}}{C_{1212}}, \\
& \bar{\alpha}_{1}=\frac{\alpha_{1}}{C_{1212}}, \\
& \bar{\rho}=\frac{\rho}{C_{1212}} .
\end{aligned}
$$

In addition:

$$
\begin{aligned}
& \square_{0}^{2}=\nabla_{12}^{2}+\beta_{2} \frac{\partial^{2}}{\partial x_{3}^{2}}-\bar{\rho} \frac{\partial^{2}}{\partial t^{2}}, \\
& \square_{p}^{2}=\nabla_{12}^{2}+\frac{1}{s_{3}^{2}} \frac{\partial^{2}}{\partial x_{3}^{2}}-\beta_{6} \frac{\partial}{\partial t}, \\
& \nabla_{12}^{2}=\frac{\partial^{2}}{\partial x_{1}^{2}}+\frac{\partial^{2}}{\partial x_{2}^{2}},
\end{aligned}
$$

with $1 / s_{3}^{2}=\bar{k}_{3} / \bar{k}_{1}=k_{3} / k_{1}$ and $\beta_{6}=\eta /\left(M k_{1}\right)$. By substituting Eq. (9) into the governing equation (Eq. (8)), two uncoupled equations governing the functions $F$ and $\chi$ give [17]:

$$
\begin{aligned}
& \beta_{2}\left[\bar{k}_{1}\left(1+\beta_{1}\right) \square_{p}^{2}\left(\square_{1}^{2} \square_{2}^{2}-\bar{\rho} \delta_{3} \frac{\partial^{2}}{\partial t^{2}} \frac{\partial^{2}}{\partial x_{3}^{2}}\right)\right. \\
& +\bar{\alpha}_{1} \bar{k}_{1} \rho_{f} \frac{\partial^{2}}{\partial t^{2}}\left(\nabla_{s 1}^{2} \square_{3}^{2}+\delta_{1} \nabla_{12}^{2} \frac{\partial^{2}}{\partial x_{3}^{2}}\right) \\
& \left.-\eta \bar{\alpha}_{1}^{2} \frac{\partial}{\partial t}\left(\nabla_{s 2}^{2} \square_{3}^{2}+\delta_{2} \nabla_{12}^{2} \frac{\partial^{2}}{\partial x_{3}^{2}}\right)\right] F=0, \\
& \square_{0}^{2} \chi=0,
\end{aligned}
$$

in which: 


$$
\begin{aligned}
\square_{1}^{2} & =\nabla_{12}^{2}+\frac{1}{s_{1}^{2}} \frac{\partial^{2}}{\partial x_{3}^{2}}-\frac{\bar{\rho}}{1+\beta_{1}} \frac{\partial^{2}}{\partial t^{2}}, \\
\square_{2}^{2} & =\nabla_{12}^{2}+\frac{1}{s_{2}^{2}} \frac{\partial^{2}}{\partial x_{3}^{2}}-\frac{\bar{\rho}}{\beta_{2}} \frac{\partial^{2}}{\partial t^{2}}, \\
\square_{3}^{2}= & \nabla_{12}^{2}+\frac{\partial^{2}}{\partial x_{3}^{2}}-\frac{\bar{\rho}}{\beta_{2}} \frac{\partial^{2}}{\partial t^{2}}, \\
\nabla_{s 1}^{2}= & \nabla_{12}^{2}+\frac{\bar{\alpha}_{3}}{\bar{\alpha}_{1} s_{3}^{2}} \frac{\partial^{2}}{\partial x_{3}^{2}}, \\
\nabla_{s 2}^{2}= & \nabla_{12}^{2}+\frac{\bar{\alpha}_{3}^{2}}{\bar{\alpha}_{1}^{2}} \frac{\partial^{2}}{\partial x_{3}^{2}}, \\
\delta_{1}= & -\frac{1}{\bar{\alpha}_{1} \bar{k}_{1} \beta_{2}}\left[\bar{\alpha}_{1} \bar{k}_{1}\left(\beta_{2}-\beta_{4}\right)+\left(\bar{\alpha}_{1} \bar{k}_{3}+\bar{\alpha}_{3} \bar{k}_{1}\right) \beta_{3}\right. \\
& \left.+\bar{\alpha}_{3} \bar{k}_{3}\left(\beta_{2}-1-\beta_{1}\right)\right] \\
\frac{1}{s_{1}^{2} s_{2}^{2}} & =\frac{1}{1+\beta_{1}} \cdot \frac{\beta_{2}^{2}-\beta_{3}^{2}+\beta_{4}\left(1+\beta_{1}\right)}{s_{2}^{2}}, \\
\delta_{3}= & \frac{1}{\bar{\alpha}_{1}^{2} \beta_{2}}\left[\bar{\alpha}_{3}^{2}\left(1+\beta_{1}-\beta_{2}\right)+\bar{\alpha}_{1}^{2}\left(\beta_{4}-\beta_{2}\right)-2 \bar{\alpha}_{1} \bar{\alpha}_{3} \beta_{3}\right] \\
s_{1} & \left(1-\frac{1}{s_{2}^{2}}\right)+\frac{1}{\beta_{2}}\left(\frac{\beta_{4}}{1+\beta_{1}}-\frac{1}{s_{1}^{2}}\right)
\end{aligned}
$$

Moreover, solving the following equation:

$$
\begin{array}{r}
C_{3333} C_{1313} s^{4}+\left(C^{2}{ }_{1133}+2 C_{1133} C_{1313}\right. \\
\left.-C_{1111} C_{3333}\right) s^{2}+C_{1111} C_{1313}=0
\end{array}
$$

the roots $s_{1}$ and $s_{2}$, which are positive and nonzero, could be obtained. In fact, $s_{1}$ and $s_{2}$ could be either real distinct or real coalescent, and or conjugate complex. Besides, in view of the Eq. (5), it is evident that when the roots are real, then they are not negative [36].

It is worth mentioning that each of the governing equations of motion and the fluid flow equation is a second-order partial differential equation in terms of special coordinates for both displacements and pore fluid pressure. In addition, they are second-order in terms of time for displacements and first order for pore fluid pressure (see Eq. (8)). Thus, in general, there is a partial differential equation of order 8 in terms of special coordinates and of order 7 in terms of time. Furthermore, the PDEs for scalar potential functions are the same. By detailed investigation of Eq. (12a), we can see that the order in special coordinates and

time is 6 and 5, respectively, and the order in Eq. (12b) is two in terms of both time and special coordinates.

Besides, one of the important features of the proposed potential functions is the physical concept of the operators used in them. Eq. (12b) is a wave equation, while Eq. (12a) contains repeated wavetransport operator as the main part of the operator applied on the potential function $F\left(x_{1}, x_{2}, x_{3}, t\right)$, and some perturbed wave operator applied on the time derivatives of $F\left(x_{1}, x_{2}, x_{3}, t\right)$. Operator $\square_{p}^{2}$ expresses the equation for the transfer of pressure in a viscous fluid (see also [17]). The viscosity parameter in this equation results in the damped response. Also, $\square_{1}^{2}$ demonstrates the propagation of longitudinal waves, while $\square_{2}^{2}$ and $\square_{3}^{2}$ show the propagation of transverse waves $($ see $[17,36])$. In addition, $\nabla_{s 1}^{2}$ and $\nabla_{s 2}^{2}$ are two different scaled Laplace operators (see also [46]). According to Eq. (9), it can be deduced that the response of the transversely isotropic saturated media is oscillatory with damping and that the engaged longitudinal (compressive) and transverse waves appear in this media.

Thus, one may search for wave functions with finite/infinite wave velocities as solutions of Eqs. (12a) and (12b). It should be noted that as soon as the time being replaced with a scalar either real or complex parameter, in the simplified $\mathbf{u}-p$ formulation, to formulate and solve the governing differential equations with the use of these potential functions will be possible. Thus, the proposed potential functions can be used in both time domain accompanied by Laplace integral transform and frequency domain. To explore the kinds of waves traveling in the domain (the body of saturated transversely isotropic poroelastic material) and their velocities, plane waves in the form of:

$$
F=A e^{i \omega\left(S x_{p} m_{p}-t\right)}, \quad \chi=B e^{i \omega\left(S x_{p} m_{p}-t\right)},
$$

are sought as solutions of Eqs. (12a) and (12b), where $i=\sqrt{-1}, \omega$ is the angular frequency of the wave and $S$ is the slowness of the plane wave traveling in the domain. $A$ and $B$ are constants showing the amplitudes of the wave induced by the scalar functions $F$ and $\chi$, respectively. In addition, $\mathbf{m}=\left(m_{1}, m_{2}, m_{3}=\cos \varphi\right)$ with $|\mathbf{m}|=1$ is the wave normal (see Figure 1 ), that identifies the direction of the propagation of plane waves. In an isotropic medium, it is known that for wave propagation in any direction, there always exist three real body waves with mutually orthogonal polarization, which coincide with the dynamic axes formed by the wavefront and propagation vector [47]. In anisotropic elastic mediums, three body waves might propagate in each direction, however, while the associated displacement vectors are mutually perpendicular, these waves cannot generally be classified into pure dilatational and rotational types [48]. One body wave 


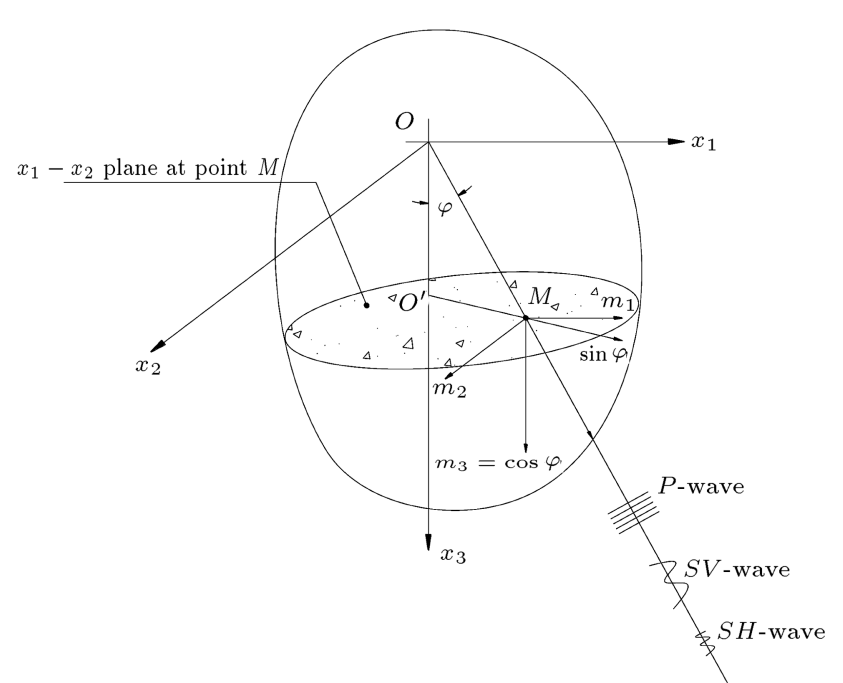

Figure 1. A schematic figure to show propagation of $P_{-}$, $S V$-, and $S H$-waves, in the direction of $\mathbf{m}$.

is always purely transverse in transversely isotropic materials, and the other two body waves are neither purely dilatational nor purely transverse type. Thus, they may be called Quasi-Longitudinal (QL) wave and Quasi-Transverse (QT) wave. If $\mathbf{u . m}=0$, then, the wave defined by Eqs. (15) is a shear (transverse) body wave. In reference to Figure 1, the shear wave propagating in $x_{1}-x_{2}$ plane with an amplitude in the same plane is called "horizontally" polarized shear wave or $S H$-wave.

By substituting the functions given in Eqs. (15) into Eqs. (12) the characteristic equations for the governing equations of the potential functions $F$ and $\chi$ are determined, respectively, as:

$$
\begin{aligned}
& c_{1} S^{6}+c_{2} S^{4}+c_{3} S^{2}+c_{4}=0, \\
& \bar{\rho}-\left(\sin ^{2} \varphi+\beta_{2} \cos ^{2} \varphi\right) S^{2}=0
\end{aligned}
$$

where $\bar{\rho}$ is given in Eqs. (10), and:

$$
\begin{aligned}
c_{1}(\omega)= & -\omega\left(\sin ^{2} \varphi+\frac{\cos ^{2} \varphi}{s_{1}^{2}}\right)\left(\sin ^{2} \varphi+\frac{\cos ^{2} \varphi}{s_{2}^{2}}\right) \\
& \left(\sin ^{2} \varphi+\frac{\cos ^{2} \varphi}{s_{3}^{2}}\right), \\
c_{2}(\omega)= & \frac{\bar{\rho} \omega}{\beta_{2}}\left(\sin ^{2} \varphi+\frac{\cos ^{2} \varphi}{s_{3}^{2}}\right)\left(\sin ^{2} \varphi+\frac{\cos ^{2} \varphi}{s_{1}^{2} s_{2}^{2}}\right) \\
+ & \beta_{6} i\left(\sin ^{2} \varphi+\frac{\cos ^{2} \varphi}{s_{1}^{2}}\right)\left(\sin ^{2} \varphi+\frac{\cos ^{2} \varphi}{s_{2}^{2}}\right) \\
+ & \frac{\bar{\rho} \omega}{1+\beta_{1}}\left(\sin ^{2} \varphi+\frac{\cos ^{2} \varphi}{s_{3}^{2}}\right)-\frac{\bar{\alpha}_{1} \rho_{f} \omega}{1+\beta_{1}}
\end{aligned}
$$

$$
\begin{aligned}
& {\left[\left(\sin ^{2} \varphi+\frac{\bar{\alpha}_{3}}{\bar{\alpha}_{1}} \frac{\cos ^{2} \varphi}{s_{3}^{2}}\right)+\delta_{1} \sin ^{2} \varphi \cos ^{2} \varphi\right] } \\
&+i \frac{\eta}{\bar{k}_{1}} \frac{\bar{\alpha}_{1}^{2}}{1+\beta_{1}}\left[\left(\sin ^{2} \varphi+\frac{\bar{\alpha}_{3}^{2}}{\bar{\alpha}_{1}^{2}} \cos ^{2} \varphi\right)\right. \\
&\left.+\delta_{2} \sin ^{2} \varphi \cos ^{2} \varphi\right] \\
& c_{3}(\omega)=-\left\{\frac{\bar{\rho} \omega}{1+\beta_{1}} \frac{\bar{\rho}}{\beta_{2}}\left(\sin ^{2} \varphi+\frac{\cos ^{2} \varphi}{s_{3}^{2}}\right)+\frac{\bar{\rho}}{1+\beta_{1}} \beta_{6} i\right. \\
&+\frac{\bar{\rho}}{\beta_{2}} \beta_{6} i\left(\sin ^{2} \varphi+\frac{\cos ^{2} \varphi}{s_{1}^{2} s_{2}^{2}}\right)-\frac{\overline{\alpha_{1}} \rho_{f} \omega}{1+\beta_{1}} \frac{\bar{\rho}}{\beta_{2}} \\
&\left.\left(\sin ^{2} \varphi+\frac{\bar{\alpha}_{3}}{\bar{\alpha}_{1}} \frac{\cos ^{2} \varphi}{s_{3}^{2}}\right)\right\}-i \frac{\eta}{\bar{k}_{1}} \frac{\bar{\rho}}{\beta_{2}} \frac{\bar{\alpha}_{1}^{2}}{1+\beta_{1}} \\
&\left(\sin ^{2} \varphi+\frac{\bar{\alpha}_{3}^{2}}{\bar{\alpha}_{1}^{2}} \cos ^{2} \varphi\right), \\
& c_{4}=\frac{\bar{\rho}}{1+}+\beta_{1} \frac{\bar{\rho}}{\beta_{2}} \beta_{6} i .
\end{aligned}
$$

Thus, it has been revealed that the body waves in fully saturated media could be separated into two secular equations described by Eqs. (16) and (17).

\section{3. $P$ - and $S V$-waves}

The secular equation (Eq. (16)) is a bi-cubic equation for the slowness, $S$, whose solution for general complex coefficients is given as:

$$
\begin{aligned}
& S_{k}^{2}(\omega)=-\frac{1}{3 c_{1}(\omega)}\left(c_{2}(\omega)+a_{k} D(\omega)+\frac{\Delta_{0}(\omega)}{a_{k} D(\omega)}\right) \\
& k=1,2,3
\end{aligned}
$$

where:

$$
\begin{aligned}
& a_{1}=1, \quad a_{2}=\frac{-1+i \sqrt{3}}{2}, \quad a_{3}=\frac{-1-i \sqrt{3}}{2} \\
& D(\omega)=\sqrt[3]{\frac{\Delta_{1}+\sqrt{\Delta_{1}^{2}-4 \Delta_{0}^{3}}}{2}} \\
& \Delta_{0}(\omega)=c_{2}^{2}-3 c_{1} c_{3} \\
& \Delta_{1}(\omega)=2 c_{2}^{3}-9 c_{1} c_{2} c_{3}+27 c_{1}^{2} c_{4} .
\end{aligned}
$$

All of the three kinds of waves with the slowness determined from Eq. (16) are attenuated, as clearly indicates from Eqs. (19) and (20). These waves are dispersive since their velocities depend on the frequency, $\omega$, as it is evident from Eq. (19). Here the slowness is no longer real. Thus the scalar potential function $F$ in 
Eq. (15) can be written more generally in the form of $A_{k} e^{-\omega \operatorname{Im}\left(S_{k}\right) x_{p} m_{p}} e^{i \omega\left[\operatorname{Re}\left(S_{k}\right) x_{p} m_{p}-t\right]}$, which shows that the propagation velocity is defined as $1 / \operatorname{Re}\left(S_{k}\right)$, while the attenuation coefficient is denoted as $\operatorname{Im}\left(S_{k}\right)$ for certain frequency. There are two dilatational waves known as dilatational waves of the first and the second kind. The waves of the first kind have very small attenuation, while the waves of the second kind are highly attenuated [8,12]. The extra compressional wave is known as slow longitudinal or $P 2$-wave since its velocity is smaller than the conventional compressional wave. On the other hand, the conventional compressional wave is referred to as the fast compressional wave or $P 1$-wave. The $P 1$ - and $S$-waves induce a motion for the fluid particles that is practically in-phase with the motion of the solid. However, the $P 2$-mode induces an out-ofphase motion for the fluid compared to the solid, which leads to its strong attenuation by the viscous damping mechanism associated with the relative fluid-solid motion. Moreover, both $P 1$ - and $S$-waves have relatively small attenuation, as mentioned for the saturated poroelastic isotropic media [12]. The slow wave has a quasi-static character at low- frequencies and becomes overdamped due to the fluid viscosity [28]. Knowing that in the absence of fluid or when the porosity is zero, there are two waves in the transversely isotropic elastic medium, namely QL and QT waves $[37,48,49]$, one can infer that the appearance of an additional wave is due to the existence of the fluid in the medium.

By further detailed investigation of Eq. (18), the governing equation (Eq. (16)) can be written as:

$$
i \omega S^{2}\left[\hat{c}_{1} S^{4}+\hat{c}_{2} S^{2}+\hat{c}_{3}\right]+\left[\hat{c}_{4} S^{4}+\hat{c}_{5} S^{2}+\hat{c}_{6}\right]=0
$$

where:

$$
\begin{aligned}
\hat{c}_{1}= & -\left(\sin ^{2} \varphi+\frac{\cos ^{2} \varphi}{s_{1}^{2}}\right)\left(\sin ^{2} \varphi+\frac{\cos ^{2} \varphi}{s_{2}^{2}}\right) \\
& \left(\sin ^{2} \varphi+\frac{\cos ^{2} \varphi}{s_{3}^{2}}\right), \\
\hat{c}_{2}= & \left(\sin ^{2} \varphi+\frac{\cos ^{2} \varphi}{s_{3}^{2}}\right)\left[\frac{\bar{\rho}}{\beta_{2}}\left(\sin ^{2} \varphi+\frac{\cos ^{2} \varphi}{s_{1}^{2} s_{2}^{2}}\right)\right. \\
& \left.+\frac{\bar{\rho}}{1+\beta_{1}}\right]-\frac{\bar{\alpha}_{1} \rho_{f}}{1+\beta_{1}}\left[\left(\sin ^{2} \varphi+\frac{\bar{\alpha}_{3}}{\bar{\alpha}_{1}} \frac{\cos ^{2} \varphi}{s_{3}^{2}}\right)\right. \\
& \left.+\delta_{1} \sin ^{2} \varphi \cos ^{2} \varphi\right], \\
\hat{c}_{3}= & -\frac{\bar{\rho}}{\beta_{2}}\left[\frac{\bar{\rho}}{1+\beta_{1}}\left(\sin ^{2} \varphi+\frac{\cos ^{2} \varphi}{s_{3}^{2}}\right)-\frac{\bar{\alpha}_{1} \rho_{f}}{1+\beta_{1}}\right. \\
& \left.\left(\sin ^{2} \varphi+\frac{\bar{\alpha}_{3}}{\bar{\alpha}_{1}} \frac{\cos ^{2} \varphi}{s_{3}^{2}}\right)\right],
\end{aligned}
$$

$$
\begin{aligned}
\hat{c}_{4}= & -\left\{\beta_{6}\left(\sin ^{2} \varphi+\frac{\cos ^{2} \varphi}{s_{1}^{2}}\right)\left(\sin ^{2} \varphi+\frac{\cos ^{2} \varphi}{s_{2}^{2}}\right)\right. \\
& +\frac{\eta}{\bar{k}_{1}} \frac{\bar{\alpha}_{1}^{2}}{1+\beta_{1}}\left[\left(\sin ^{2} \varphi+\frac{\bar{\alpha}_{3}^{2}}{\bar{\alpha}_{1}^{2}} \cos ^{2} \varphi\right)\right. \\
& \left.\left.+\delta_{2} \sin ^{2} \varphi \cos ^{2} \varphi\right]\right\} \\
\hat{c}_{5}= & \beta_{6}\left[\frac{\bar{\rho}}{1+\beta_{1}}+\frac{\bar{\rho}}{\beta_{2}}\left(\sin ^{2} \varphi+\frac{\cos ^{2} \varphi}{s_{1}^{2} s_{2}^{2}}\right)\right] \\
& +\frac{\eta}{\bar{k}_{1}} \frac{\bar{\rho}}{\beta_{2}} \frac{\bar{\alpha}_{1}^{2}}{1+\beta_{1}}\left(\sin ^{2} \varphi+\frac{\bar{\alpha}_{3}^{2}}{\bar{\alpha}_{1}^{2}} \cos ^{2} \varphi\right) \\
\hat{c}_{6}= & -\beta_{6} \frac{\bar{\rho}}{1+\beta_{1}} \frac{\bar{\rho}}{\beta_{2}} .
\end{aligned}
$$

As it is evident in the low-frequency limit $(\omega \rightarrow 0)$, the secular equation (Eq. (21)) takes the form:

$$
\hat{c}_{4} S^{4}+\hat{c}_{5} S^{2}+\hat{c}_{6}=0 .
$$

It is convenient to denote the solution of Eq. (23) by $S_{1(\omega=0)}$ and $S_{2(\omega=0)}$. Biot [8] showed that both the phase velocity and the attenuation of the wave of the second kind tend to zero as the frequency, $\omega$, approaches zero. Also, attenuation coefficients of $P 1$ - and $S V$-waves are zero at zero-frequency. So, these roots, which are obtained by Eq. (23), are not dispersive and correspond to the velocity of the first kind and the rotational waves.

\section{4. $S H$-wave}

We now wish to investigate the waves induced by the function $\chi$, in more detail. Eq. (17) has one root which is generally different from any of the three roots of Eq. (16). By virtue of Eq. (17), one could result in that:

$$
S=\sqrt{\frac{\rho}{C_{1212} \sin ^{2} \varphi+C_{1313} \cos ^{2} \varphi}} .
$$

Similar to a transversely isotropic dry elastic medium, the displacement due to $\chi$-function is related to $S H$ wave. From the linearity of Eq. (8) with respect to $u_{i}$ and Eq. (9) with respect to the potential functions $F$ and $\chi$, it can be concluded that the displacement field corresponds only to the function $\chi$, in the form of:

$$
\mathbf{u}=\left(-\frac{\partial \chi}{\partial x_{2}}, \frac{\partial \chi}{\partial x_{1}}, 0\right)
$$

This equation implies that the displacement vector has no component in the $x_{3}$-direction. The conventional inner product of the unit vectors of the direction of 
propagation and the displacement vector, which may be expressed as:

$$
\mathbf{u} . \mathbf{m}=\left(-i \omega S m_{2} \chi, i \omega S m_{1} \chi, 0\right) \cdot\left(m_{1}, m_{2}, m_{3}\right)=0
$$

indicates that the displacement vector and the wavenormal are perpendicular, namely the displacement is polarized in the $x_{1}-x_{2}$ plane. Therefore, this solution describes a pure shear wave, so, based on our early description, this solution implies that the function $\chi$ produces a pure $S H$-wave. So, we can write from Eq. (24) that $v_{S H}=\sqrt{\left(C_{1212} \sin ^{2} \varphi+C_{1313} \cos ^{2} \varphi\right) / \rho}$. As it is observed, the velocity of the $S H$-wave is determined from an equation which is the same as the formulation given for a dry (one-phase) solid part, except that the mass density is the bulk mass density of the saturated porous medium. Consequently, the behavior of $S H$-wave in saturated porous materials is similar to its behavior in the single-phase.

\section{Special cases}

As indicated in this paper, in general, four different body waves could travel in transversely isotropic fully saturated poroelastic media. As indicated in Eq. (19), the slowness (velocity) of different body waves in a saturated transversely isotropic poroelastic material depends on the material properties of fluid and solid, and the direction of propagation as well. In this section, the slowness determined in previous sections is expressed for some special direction of propagation and is also simplified for some special cases of material filling the domain.

As shown in Figure 1, if $\varphi=0$, the wave propagates along the axis of material symmetry of the transversely isotropic material. By putting $\varphi=0$ in Eq. (19), the slowness of the wave induced by the potential function $F$ is determined. To do so, first, the parameters $c_{k}$ given in Eq. (18) are simplified for $\varphi=0$ as:

$$
\begin{aligned}
& c_{1}(\omega)= i \omega, \\
& c_{2}(\omega)=-i \omega\left(\frac{\rho-\alpha_{3} \rho_{f}}{C_{3333}}+\frac{\rho}{C_{1313}}\right) \\
&+\frac{\eta}{k_{3}}\left(\frac{1}{M}+\frac{\alpha_{3}^{2}}{C_{3333}}\right),
\end{aligned}
$$

$$
\begin{aligned}
& c_{3}(\omega)=-i \omega \frac{\rho}{C_{1313}} \frac{\rho-\alpha_{3} \rho_{f}}{C_{3333}}-\rho \frac{\eta}{k_{3}} \\
& {\left[\frac{1}{M}\left(\frac{1}{C_{3333}}+\frac{1}{C_{1313}}\right)+\frac{1}{C_{1313}} \frac{\alpha_{3}^{2}}{C_{3333}}\right]} \\
& c_{4}=\frac{\rho}{C_{3333}} \frac{\rho}{C_{1313}} \frac{1}{M} \frac{\eta}{k_{3}} .
\end{aligned}
$$

The rational root may also be used for a cubic equation with a rational coefficient. In this way, the slowness of $P 1$ - and $P 2$-waves are given in Eq. (28) as shown in Box II, while the slowness of the $S V$-wave is given by:

$$
S_{S V}=\sqrt{\frac{\rho}{C_{1313}}} .
$$

The slowness given in Eq. (28) shows that in this case, the longitudinal wave velocities and corresponding attenuation coefficients depend on those properties of solid and liquid, which directly describe the behavior in the direction of the axis of symmetry, i.e. $C_{3333}$, $\alpha_{3}$, and $k_{3}$. Also, $S_{P 1}$ and $S_{P 2}$ are dispersive, while the transverse wave velocity is found to be purely transverse, which propagates without dispersion with a velocity which is dependent on the shear elasticity coefficient. In addition, $S_{P 1(\omega=0)}=\sqrt{\rho / A_{3333}}$ and $S_{P 2(\omega=0)}$ is zero.

Also, the wave incident is perpendicular to the axis of symmetry and propagates in a horizontal direction when $\varphi=\pi / 2$ (Figure 1$)$. In this case:

$$
\begin{aligned}
& c_{1}(\omega)= i \omega, \\
& c_{2}(\omega)=-i \omega\left(\frac{\rho-\alpha_{1} \rho_{f}}{C_{1111}}+\frac{\rho}{C_{1313}}\right) \\
&+\frac{\eta}{k_{1}}\left(\frac{1}{M}+\frac{\alpha_{1}^{2}}{C_{1111}}\right), \\
& c_{3}(\omega)= i \omega \frac{\rho}{C_{1313}} \frac{\rho-\alpha_{1} \rho_{f}}{C_{1111}}-\rho \frac{\eta}{k_{1}} \\
& \quad\left[\frac{1}{M}\left(\frac{1}{C_{1111}}+\frac{1}{C_{1313}}\right)+\frac{1}{C_{1313}} \frac{\alpha_{1}^{2}}{C_{1111}}\right], \\
& c_{4}=\frac{\rho}{C_{1111}} \frac{\rho}{C_{1313}} \frac{1}{M} \frac{\eta}{k_{1}},
\end{aligned}
$$

and the slowness can be obtained from Eqs. (19) and

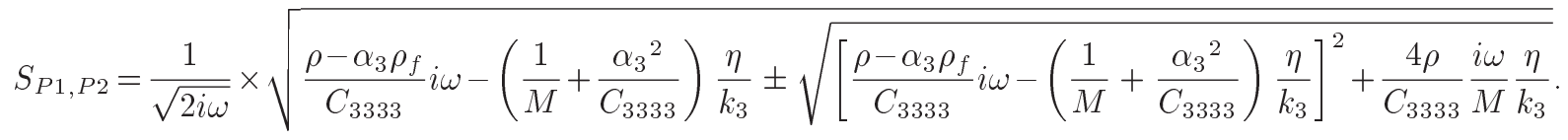




$$
\begin{aligned}
& S_{P 1, P 2}=\frac{1}{\sqrt{2 i \omega}} \times \sqrt{\frac{\rho-\alpha_{1} \rho_{f}}{C_{1111}} i \omega-\left(\frac{1}{M}+\frac{\alpha_{1}^{2}}{C_{1111}}\right) \frac{\eta}{k_{1}} \pm \sqrt{\left[\frac{\rho-\alpha_{1} \rho_{f}}{C_{1111}} i \omega-\left(\frac{1}{M}+\frac{\alpha_{1}{ }^{2}}{C_{1111}}\right) \frac{\eta}{k_{1}}\right]^{2}+\frac{4 \rho}{C_{1111}} \frac{i \omega}{M} \frac{\eta}{k_{1}}}} \\
& S_{S V}=\sqrt{\frac{\rho}{C_{1313}}}
\end{aligned}
$$

$$
\begin{aligned}
& S_{P 1, P 2}=\frac{1}{\sqrt{2 i \omega}} \times \sqrt{\frac{\rho-\alpha \rho_{f}}{\lambda+2 \mu} i \omega-\left(\frac{1}{M}+\frac{\alpha^{2}}{\lambda+2 \mu}\right) \frac{\eta}{k} \pm \sqrt{\left[\frac{\rho-\alpha \rho_{f}}{\lambda+2 \mu} i \omega-\left(\frac{1}{M}+\frac{\alpha^{2}}{\lambda+2 \mu}\right) \frac{\eta}{k}\right]^{2}+\frac{4 \rho}{\lambda+2 \mu} \frac{i \omega}{M} \frac{\eta}{k}}} \\
& S_{S V}=\sqrt{\frac{\rho}{\mu}}
\end{aligned}
$$

Box IV

(20). Similar to the previous case, here the rational root test is helpful, so it can be explicitly shown that the slowness is obtained by Eq. (31) as shown in Box III.

Thus, $P 1$ - and $P 2$-waves velocities and related attenuation coefficients, in this case, are both related to material properties in a plane perpendicular to the axis of symmetry. As seen previously, the $S V$-wave velocity is written in terms of shear modulus. Finally, when $\omega=0$, one may find $S_{P 1(\omega=0)}=\sqrt{\rho / A_{1111}}$, while $S_{P 2(\omega=0)}$ is disappeared.

$S H$-wave slowness can be computed for $\varphi=0$ and $\varphi=\pi / 2$, from Eq. (24) as $\sqrt{\rho / C_{1313}}$ and $\sqrt{\rho / C_{1212}}$, respectively. As it is observed, these waves are pure transverse waves.

In addition, the material properties, in a special case in which the transmitting material is isotropic in both hydraulic and mechanical points of view, are written as:

$$
\begin{aligned}
& C_{1111}=C_{3333}=\lambda+2 \mu, \quad C_{1122}=C_{1133}=\lambda, \\
& C_{1313}=\mu, \quad \alpha_{1}=\alpha_{3}=\alpha, \quad k_{1}=k_{3}=k,
\end{aligned}
$$

where $\lambda$ and $\mu$ are Lame's constants. We then found that $s_{1}^{2}=s_{2}^{2}=1$. In this case, it can be easily shown that the parameters $\delta_{1}, \delta_{2}$ and $\delta_{3}$ are identically zero and $s_{3}^{2}=1$. By substituting these values into Eq. (16), and applying the rotational root test, the solution of the governing secular equation leads to Eq. (33) as shown in Box IV.

With the aid of Eq. (24), for isotropic elastic materials, it can be deduced that:

$$
S_{S H}=\sqrt{\frac{\rho}{\mu}} .
$$

In addition, in this case:

$$
S_{P 1(\omega=0)}=\sqrt{\rho /\left[(\lambda+2 \mu)+\alpha^{2} M\right]} .
$$

Besides the general model, where all constituents are compressible, an idealized version with incompressible constituents can also be considered. When both constituents are incompressible, i.e. $K / K_{s} \ll 1$ and $K / K_{f} \ll 1$, these results may be obtained: $\alpha_{1}=\alpha_{3}=$ 1 and $M \rightarrow \infty[50,51]$. Inserting these conditions in the material properties, an infinite wave speed for the fast compressional wave could be obtained, while the slowness of other waves changes to:

for transversely isotropic material and $\varphi=0$ :

$$
S_{P 2}=\sqrt{\frac{1}{C_{3333} i \omega}\left[\left(\rho-\rho_{f}\right) i \omega-\frac{\eta}{k_{3}}\right]}
$$

for transversely isotropic material and $\varphi=\frac{\pi}{2}$ :

$$
S_{P 2}=\sqrt{\frac{1}{C_{1111} i \omega}\left[\left(\rho-\rho_{f}\right) i \omega-\frac{\eta}{k_{1}}\right]},
$$

for isotropic material:

$$
S_{P 2}=\sqrt{\frac{1}{(\lambda+2 \mu) i \omega}\left[\left(\rho-\rho_{f}\right) i \omega-\frac{\eta}{k}\right]},
$$

and by virtue of Eq. (23):

$$
S_{S V(\omega=0)}=\sqrt{\bar{\rho} /\left[\beta_{2}\left(1+\delta_{2} \sin ^{2} \varphi \cos ^{2} \varphi\right)\right]} .
$$


To prove the validity of the results presented here, we examined the results for a single-phase elastic medium, and compared them with the formulations reported in the literature. To define a single-phase, the porosity $n$ must be zero. As a result $\alpha_{1}=\alpha_{3}=0$ and $M \rightarrow \infty$. Therefore, the parameters $\bar{\alpha}_{1}, \bar{\alpha}_{3}, \beta_{5}$ and $\beta_{6}$ are identically zero. It can be easily deduced that the coefficient in Eq. (18d) is neglected. Consequently, the secular equation (Eq. (16)) leads to $S^{2}\left(c_{1} S^{4}-c_{2} S^{2}+\right.$ $1)=0$. As it is observed, in this case, one of the roots of Eq. (16) is zero, while the others are the roots of the quadratic equation:

$$
c_{1} S^{4}-c_{2} S^{2}+1=0
$$

where:

$$
\begin{aligned}
& c_{1}=\frac{\beta_{2}}{\bar{\rho}} \frac{1+\beta_{1}}{\bar{\rho}}\left(\sin ^{2} \varphi+\frac{\cos ^{2} \varphi}{s_{1}^{2}}\right)\left(\sin ^{2} \varphi+\frac{\cos ^{2} \varphi}{s_{2}^{2}}\right), \\
& c_{2}=\frac{1+\beta_{1}}{\bar{\rho}}\left(\sin ^{2} \varphi+\frac{\cos ^{2} \varphi}{s_{1}^{2} s_{2}^{2}}\right)+\frac{\beta_{2}}{\bar{\rho}} .
\end{aligned}
$$

The slowness in this case is:

$$
\begin{aligned}
S_{P 1} & =\frac{1}{v_{P 1}} \\
& =\sqrt{\frac{2 \rho_{s}}{C_{1111} \sin ^{2} \varphi+C_{3333} \cos ^{2} \varphi+C_{1313}+\sqrt{\Omega}}}, \\
S_{S V} & =\frac{1}{v_{S V}} \\
& =\sqrt{\frac{2 \rho_{s}}{C_{1111} \sin ^{2} \varphi+C_{3333} \cos ^{2} \varphi+C_{1313}-\sqrt{\Omega}}}, \\
\Omega= & {\left[\left(C_{1111}-C_{1313}\right) \sin ^{2} \varphi-\left(C_{3333}-C_{1313}\right) \cos ^{2} \varphi\right]^{2} } \\
& +\left(C_{1133}+C_{1313}\right)^{2} \sin ^{2} 2 \varphi .
\end{aligned}
$$

Moreover:

$$
S_{S H}=\frac{1}{v_{S H}}=\sqrt{\frac{\rho_{s}}{C_{1212} \sin ^{2} \varphi+C_{1313} \cos ^{2} \varphi}},
$$

which have been previously derived in $[37,49]$. In general, anisotropic dry media have three body waves propagating with velocities, which are the function of the direction of phase propagation. Besides, for a particular direction of phase propagation, their polarizations are fixed and orthogonal. The waves are called quasi-waves as polarizations may not be along the wavefront and propagation vector namely dynamic axis [49].

Eventually, the velocities for isotropic singlephase material can be concluded from Eqs. (33) and (34) or (38) and (39), where one may find $v_{P 1}=$ $\sqrt{(\mu+2 \mu) / \rho_{s}}, v_{S V}=\sqrt{\mu / \rho_{s}}$, and $v_{S H}=\sqrt{\mu / \rho_{s}}$.

\section{Numerical results}

We now, in this section, present some numerical evaluations for body wave velocities and corresponding attenuation coefficients for different synthetic poroelastic transversely isotropic materials based on numerical values of the coefficients given in Table 1 . The solid density equals $\rho_{s}=2700 \mathrm{~kg} / \mathrm{m}^{3}$ and the bulk modulus of the solid is $K_{s}=1.1 \times 10^{10} \mathrm{~N} / \mathrm{m}^{2}$. The elastic parameters of the materials have been selected so that the positive definite conditions of strain energy function are satisfied in accordance with Eq. (5). The synthetic materials listed in Table 1 have been selected, such that a wide variety of degrees of anisotropy defined as $E / E^{\prime}$ and $G / G^{\prime}$ be considered in the numerical study. In

\begin{tabular}{|c|c|c|c|c|c|c|c|c|c|c|c|}
\hline Material No. & $\begin{array}{c}E \\
\left(\mathrm{~N} / \mathrm{m}^{2}\right) \\
\times 10^{7} \\
\end{array}$ & $\begin{array}{c}\boldsymbol{E}^{\prime} \\
\left(\mathrm{N} / \mathbf{m}^{2}\right) \\
\times \mathbf{1 0}^{\mathbf{7}} \\
\end{array}$ & $\begin{array}{c}G \\
\left(\mathrm{~N} / \mathrm{m}^{2}\right) \\
\times 10^{\mathbf{7}} \\
\end{array}$ & $\begin{array}{c}G^{\prime} \\
\left(\mathrm{N} / \mathrm{m}^{2}\right) \\
\times 10^{\mathbf{7}} \\
\end{array}$ & $\nu$ & $\nu^{\prime}$ & $\begin{array}{c}C_{1111} \\
\left(\mathrm{~N} / \mathrm{m}^{2}\right) \\
\times 10^{7} \\
\end{array}$ & $\begin{array}{c}C_{1133} \\
\left(\mathrm{~N} / \mathrm{m}^{2}\right) \\
\times 10^{7} \\
\end{array}$ & $\begin{array}{c}C_{\mathbf{3 3 3 3}} \\
\left(\mathrm{N} / \mathrm{m}^{2}\right) \\
\times \mathbf{1 0}^{\mathbf{7}} \\
\end{array}$ & $\begin{array}{c}C_{1313} \\
\left(\mathrm{~N} / \mathrm{m}^{2}\right) \\
\times 10^{7}\end{array}$ & $\begin{array}{c}C_{1212} \\
\left(\mathrm{~N} / \mathrm{m}^{2}\right) \\
\times 10^{7}\end{array}$ \\
\hline 1 & 5 & 5 & 2 & 2 & 0.25 & 0.25 & 6 & 2 & 6 & 2 & 2 \\
\hline 2 & 10 & 5 & 4 & 2 & 0.25 & 0.25 & 14 & 5 & 7.5 & 2 & 4 \\
\hline 3 & 5 & 5 & 2 & 1 & 0.25 & 0.25 & 6 & 2 & 6 & 1 & 2 \\
\hline 4 & 5 & 10 & 4 & 2 & 0.25 & 0.25 & 5.64 & 1.82 & 10.91 & 2 & 4 \\
\hline 5 & 10 & 5 & 2 & 2 & 0.25 & 0.25 & 14 & 5 & 7.5 & 2 & 2 \\
\hline 6 & 15 & 5 & 6 & 2 & 0.25 & 0.25 & 26 & 10 & 10 & 2 & 6 \\
\hline
\end{tabular}
this study $E$ and $E^{\prime}$ are the Young's modulus in any direction in an isotropic plane and a direction normal to it, respectively. Also, $G$ is the shear modulus in an isotropic plane, and $G^{\prime}$ is the same function in any plane parallel to the material axis of symmetry. Furthermore, $\nu$ is the Poisson's ratio related to any two perpendicular directions in horizontal plane, and $\nu^{\prime}$ is the same parameter corresponding to any direction in

Table 1. Elasticity coefficients of synthetic transversely isotropic materials. 
a horizontal plane with respect to a vertical direction. Based on this idea, Material No. 1 denoted as Mat \#1 is selected to be isotropic as a reference. Mat \#2 is a transversely isotropic material with $E / E^{\prime}=2$ and $G / G^{\prime}=2$, Mat \#3 is a transversely isotropic material with $E=E^{\prime}$ and $G / G^{\prime}=2$. Mat \#4 is a transversely isotropic material with $E / E^{\prime}=0.5$ and $G / G^{\prime}=2$. On the other hand, Mat \#5 is a transversely isotropic material with $E / E^{\prime}=2$ and $G=G^{\prime}$. Eventually, Mat \#6 is a transversely isotropic material with $E / E^{\prime}=3$ and $G / G^{\prime}=3$. As mentioned earlier, since simplification is made based on the original formulation of Biot, the range of validity of the solutions should be considered. Therefore, we, after Biot [8], suppose that the poiseuille flow breaks down for frequency larger than $f_{t}=100 \mathrm{~Hz}$. The material properties of the fluid for numerical computation are given in Table 2. The data of these coefficients have been provided based on the information given by the works of $[2,52]$. The intrinsic permeability coefficients may also be observed in Table 2. It should be noted that the intrinsic permeability considered for the materials is sufficiently small that the frequency of excitation falls within the low-frequency range. Biot's effective stress coefficient in horizontal and vertical planes and Biot's modulus can be obtained from Eqs. (6) and (7), respectively, and based on the data given in Tables 1 and 2 . In the present study, the physical quantities of interest express the influence of the type of material anisotropy, phase angle, frequency-dependent, permeability, and porosity on the velocity of propagation and corresponding attenuation coefficient.

Wang et al. [49] derived analytical solutions for body wave velocities and the direction of propagation of a continuously inhomogeneous cross-anisotropic material. In this study, the closed-form solutions were governed by the inhomogeneous parameter, the degree and type of material anisotropy and the phase angle. In addition, Raoofian-Naeeni and Eskandari-Ghadi [37] determined the longitudinal and transverse wave velocities in explicit forms for transversely isotropic media. To compare the results for a single-phase case, the solutions presented in [37] and in [49] for transversely isotropic homogeneous materials are used as benchmarks. The velocities are equal to the values, which have been presented in Eqs. (38) and (39). As indicated in Figures 2 to 4, there is an excellent agreement between the two solutions. Computations for the case $n=0$ indicate that the slowness of longitudinal

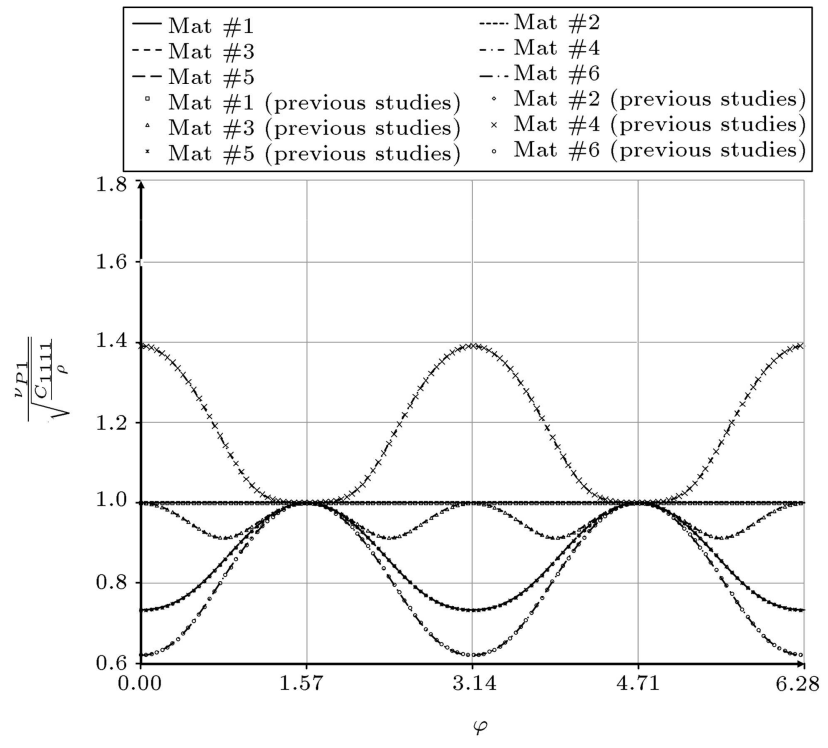

Figure 2. Comparison of normalized longitudinal $P 1$-wave velocity obtained from the present study with $[37,49]$.

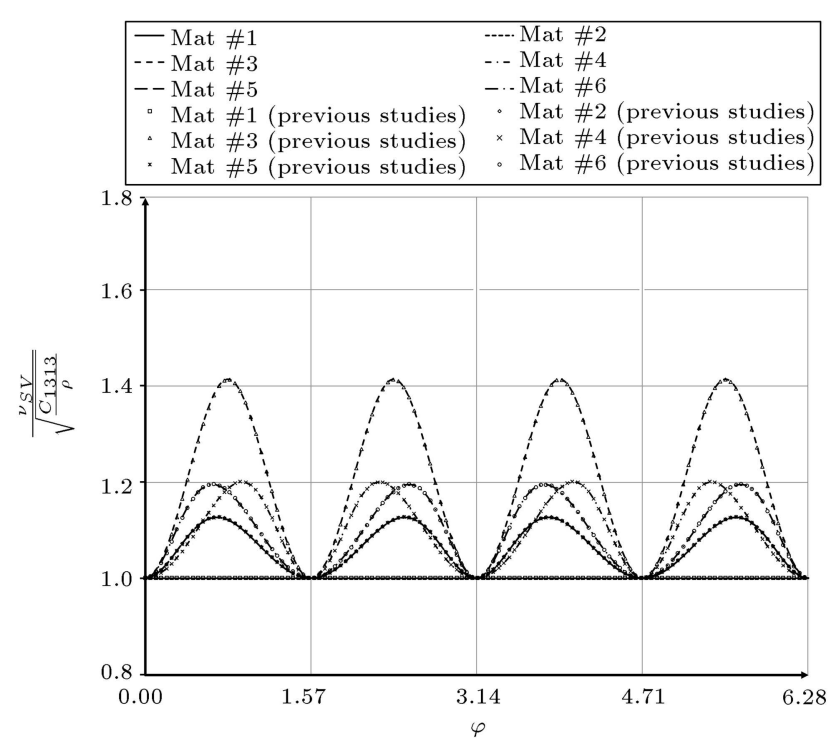

Figure 3. Comparison of normalized $S V$-wave velocity obtained from the present study with $[37,49]$.

$P 2$-wave disappears, and those of $P 1-, S V$-, and $S H$ waves are associated with the single-phase model.

Figure 5 shows the variation of the wave propagation velocity versus frequency for different values of the porosity, i.e., $n=0.2,0.5$, and 0.8 for Mat $\# 4$ and at $\varphi=\pi / 2$. As stated previously $\nu_{P 1}, \nu_{P 2}$ and $\nu_{S V}$ correspond to quasi-dilatational and quasi-

Table 2. Material data for fluid and intrinsic permeability coefficients [2,52].

\begin{tabular}{ccccc}
\hline $\boldsymbol{\rho}_{\boldsymbol{f}}\left(\mathrm{kg} / \mathbf{m}^{\mathbf{3}}\right)$ & $\boldsymbol{K}_{\boldsymbol{f}}\left(\mathrm{N} / \mathbf{m}^{\mathbf{2}}\right)$ & $\boldsymbol{k}_{\mathbf{1}}\left(\mathbf{m}^{\mathbf{2}}\right)$ & $\boldsymbol{k}_{\mathbf{3}}\left(\mathbf{m}^{\mathbf{2}}\right)$ & $\boldsymbol{\eta}\left(\mathrm{N} . \mathbf{s e c} / \mathbf{m}^{\mathbf{2}}\right)$ \\
\hline 1000 & $3.3 \times 10^{9}$ & $3.55 \times 10^{-12}$ & $3.55 \times 10^{-13}$ & $10^{-3}$ \\
\hline
\end{tabular}




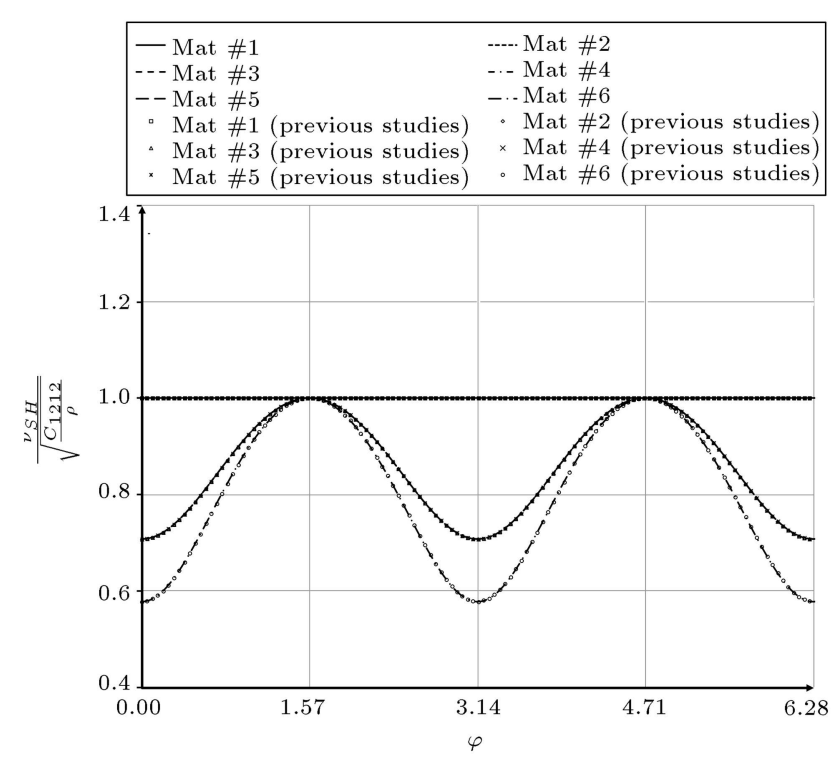

Figure 4. Comparison of normalized $S H$-wave velocity obtained from the present study with $[37,49]$.

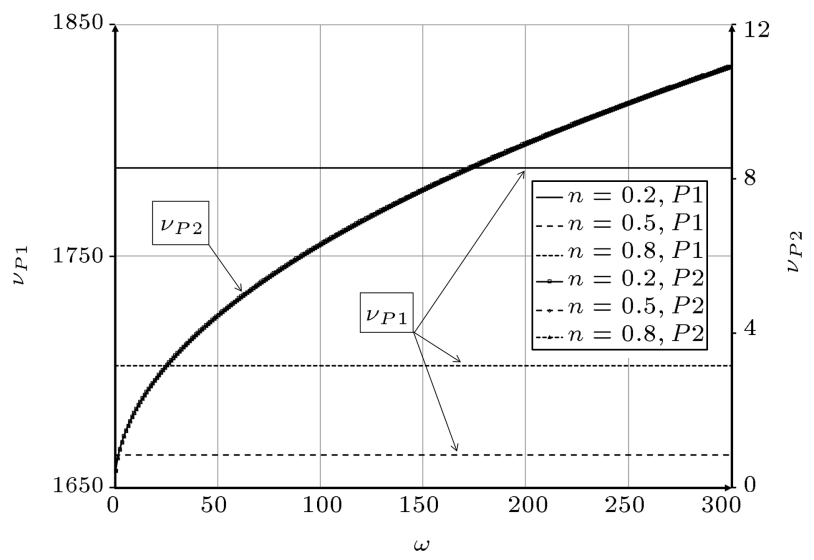

Figure 5a. $P 1$ - and $P 2$-wave velocities $(\mathrm{m} / \mathrm{sec})$ versus frequency $(\mathrm{rad} / \mathrm{sec})$ for Mat \#4, and $\varphi=\pi / 2$.

shear wave velocities, respectively. It is clear from Figure 5 that $v_{P 1}>v_{S V}>v_{P 2}$. Also, the behavior of $\nu_{P 1}$ and $\nu_{S V}$ is independent of frequency in this case, which means that they are non-dispersive. Moreover, as mentioned, $\nu_{P 1}$ and $\nu_{S V}$ for $\omega=0$ and $\varphi=\pi / 2$ equal to $\sqrt{A_{1111} / \rho}$ and $\sqrt{C_{1313} / \rho}$, respectively. Thus, based on Figures $5 \mathrm{a}$ and $5 \mathrm{~b}$, these velocities are always equal to $\sqrt{A_{1111} / \rho}$, and $\sqrt{C_{1313} / \rho}$, respectively. It should be noticed that in this expression $\rho$ is a function of the porosity, $n$, as stated earlier in Section 2. In addition, the dispersive character of the $P 2$-wave can be observed from Figure 5a. It is clear from this figure that the dependency of compressional wave velocity of the second kind on porosity for all frequencies is negligible. It is depicted that $\nu_{P 2}$ tends to be disappeared at zero frequency, as it has been analytically discussed in Section 3. As it can be observed, the lower the frequency, the higher the velocity change

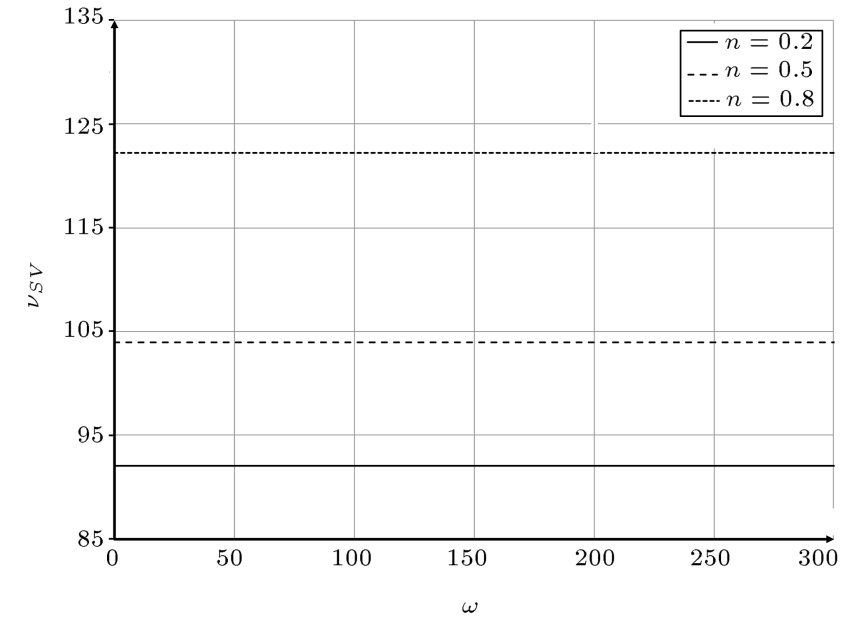

Figure 5b. $S V$-wave velocity $(\mathrm{m} / \mathrm{sec})$ versus frequency $(\mathrm{rad} / \mathrm{sec})$ for Mat $\# 4$, and $\varphi=\pi / 2$.

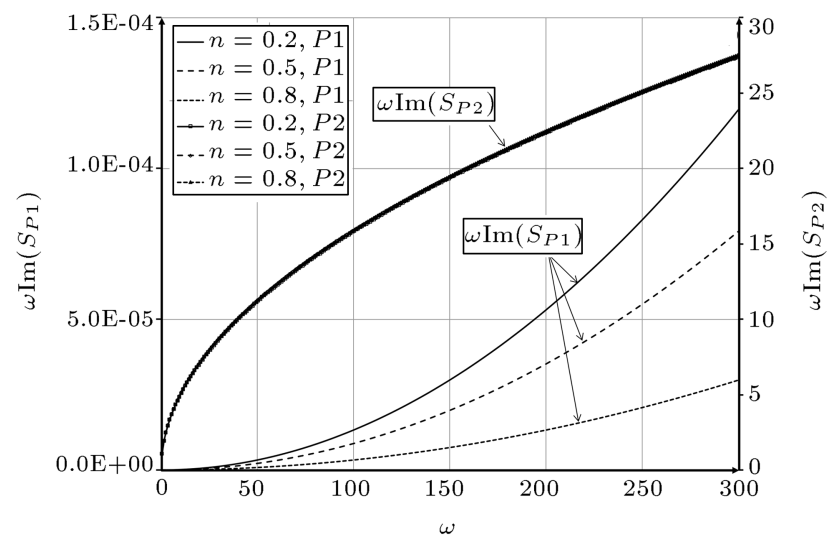

Figure 6. Attenuation coefficient of $P 1$ - and $P 2$-waves $(\mathrm{rad} / \mathrm{m})$ versus frequency $(\mathrm{rad} / \mathrm{sec})$ for Mat \#4, and $\varphi=\pi / 2$.

happens (see Figure 5a). It should also be pointed out that based on Eq. (24), $\nu_{S H}$ is independent of the frequency.

Figure 6 describes the variation of wave attenuation (see Section 3) versus $\omega$ for the specified values of $n$ for Mat \#4 and incident angle $\varphi=\pi / 2$. From Figure 6 , we can see that the $P 1$-wave attenuation coefficient is not affected by porosity in low-frequency. However, the attenuation coefficient is highly changed at highfrequency. In addition, the higher the porosity, the lower the wave attenuation. As it is clear from this figure, the attenuation coefficient of the $P 2$-wave is not affected by the level of porosity, while it is influenced by the frequency in such a way that the larger the frequency the larger the attenuation of compressional wave ( $P 2$-wave). It should be noted that according to Eq. (31), the attenuation coefficient for the transverse shear wave vanishes for incident angle equal to $\pi / 2$, and that is why the attenuation coefficient for $S V$-wave is not plotted. Also, it is seen from Figures 5 and 6 


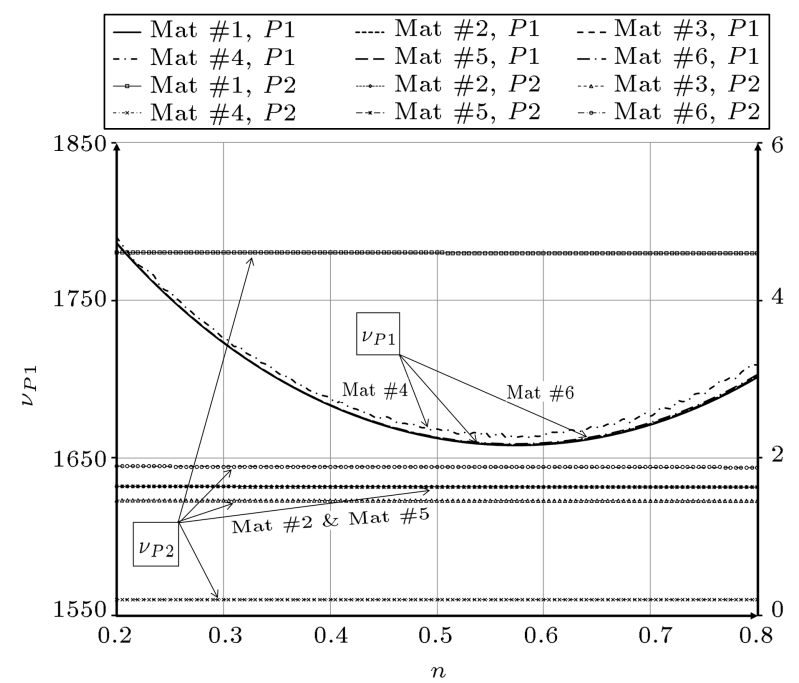

Figure 7a. $P 1$ - and $P 2$-wave velocities $(\mathrm{m} / \mathrm{sec})$ versus porosity, $\varphi=0$ and $\omega=50 \mathrm{rad} / \mathrm{sec}$.

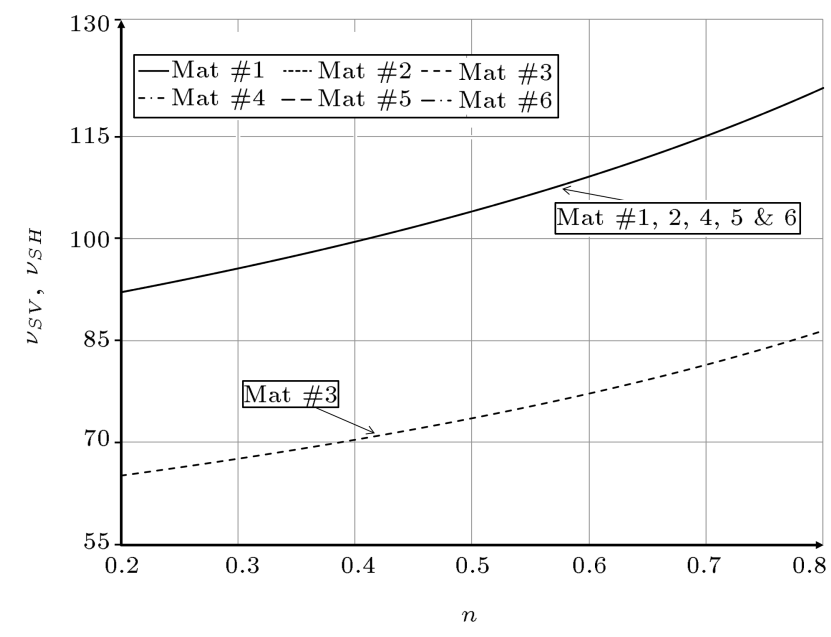

Figure 7 b. $S V$ - and $S H$-wave velocities $(\mathrm{m} / \mathrm{sec})$ versus porosity, $\varphi=0$ and $\omega=50 \mathrm{rad} / \mathrm{sec}$.

that the form of the curves is comparable to the one corresponding to Biot's model for isotropic saturated porous media.

Figures 7 and 8 provide the variation of body wave velocities and corresponding attenuation coefficients versus porosity for all materials, when $\varphi=0$. The effect of the porosity on the velocities for compressional and shear waves is depicted in Figure 7 . With the aid of numerical results, it can be shown that the trend of $\nu_{P 1}$ and $\sqrt{A_{3333} / \rho}$ at $\varphi=0$ are alike versus porosity. So, the compressional wave velocity $\nu_{P 1}$ for Mat \#4 is the largest compared with the others, which is related to the elasticity coefficient $C_{3333}$ (see Figure 7a). Inspection of Eqs. (24) and (29) indicates that the magnitudes and the variation of $\nu_{S V}$ and $\nu_{S H}$ are the same as those presented in this special case $(\varphi=0)$, and equal to $\sqrt{C_{1313} / \rho}$. On the other hand, the mass density of the mixture is influenced by the

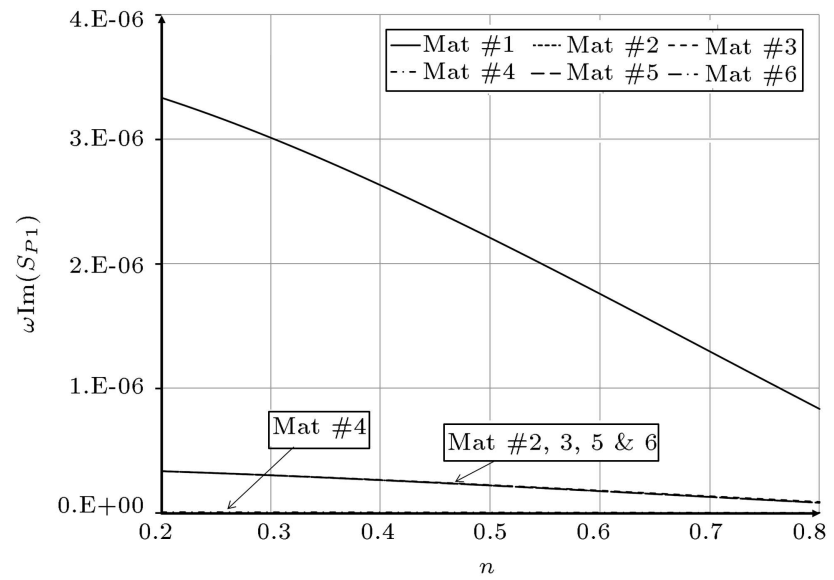

Figure 8a. $P 1$-wave attenuation coefficient $(\mathrm{rad} / \mathrm{m})$ versus porosity, $\varphi=0$, and $\omega=50 \mathrm{rad} / \mathrm{sec}$.

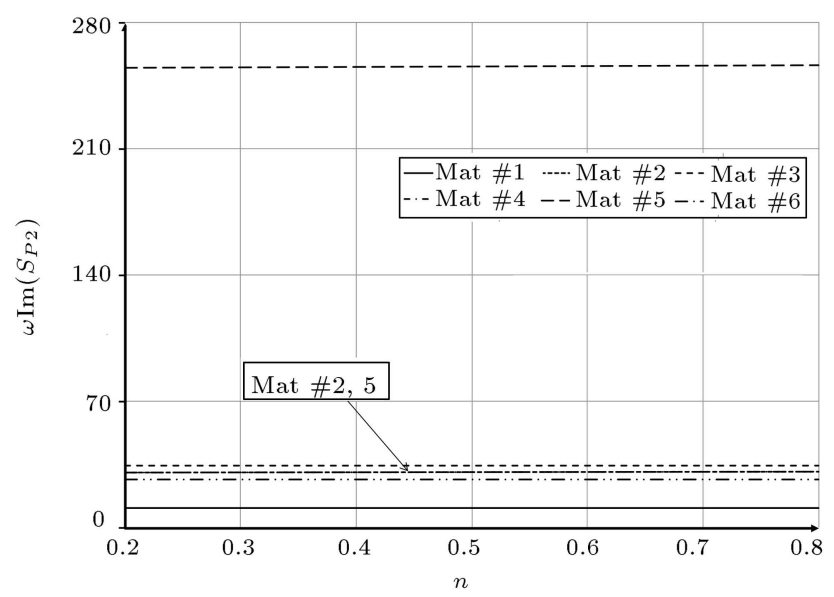

Figure 8 b. $P 2$-wave attenuation coefficient $(\mathrm{rad} / \mathrm{sec})$ versus porosity, $\varphi=0$, and $\omega=50 \mathrm{rad} / \mathrm{sec}$.

porosity, resulting in some variation for $\nu_{S V}$ and $\nu_{S H}$ in terms of the porosity. Figure $7 \mathrm{~b}$ shows the variation of $\nu_{S V}$ and $\nu_{S H}$ in terms of porosity. As could be seen, the transverse wave velocities for Mat \# 1, 2, 4, 5, and 6 are equal, since $C_{1313}$ for these materials are the same. Furthermore, the attenuation coefficient, $\omega \operatorname{Im}\left(S_{P 1}\right)$, decreases with an increase of porosity, especially in the isotropic material (Figure 8a). Also, as it is observed in Figures $7 \mathrm{a}$ and $8 \mathrm{~b}$, for the compressional wave of the second kind, the dependence of velocity and attenuation coefficient differs from materials with different anisotropy, while the effect of porosity on these parameters is negligible. Detailed investigation of Figures $7 \mathrm{a}$ and $8 \mathrm{~b}$ shows that the velocity and attenuation coefficient of $P 2$-wave for all materials are significantly different, while for Mat \#2 and Mat \#5 they are not very different. This could be due to the similarity between these two materials. On the other hand, the elasticity coefficient $C_{1212}$ for these materials was different, however, the effect of $C_{1212}$ on either $\nu_{P 2}$ or $\omega \operatorname{Im}\left(S_{P 2}\right)$ is not significant. 


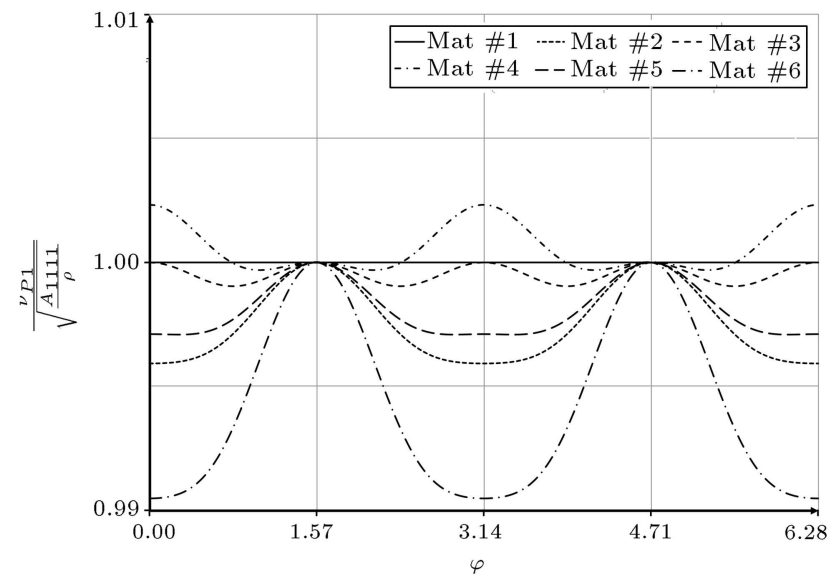

Figure 9a. Normalized $P 1$-wave velocity versus incidence angle, $n=0.5$, and $\omega=50 \mathrm{rad} / \mathrm{sec}$.

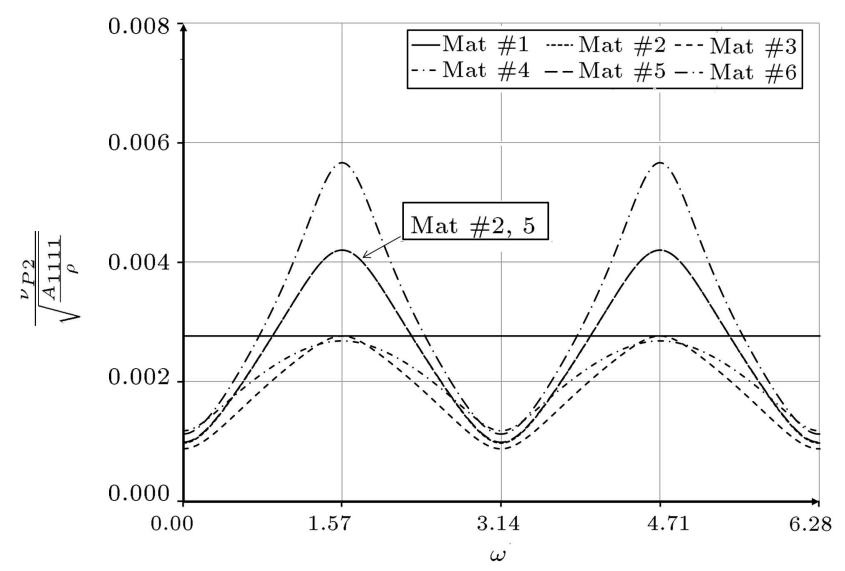

Figure 9b. Normalized $P 2$-wave velocity versus incidence angle, $n=0.5$, and $\omega=50 \mathrm{rad} / \mathrm{sec}$.

Figure 9 illustrates a graphical representation for $P 1-, P 2-, S V$-, and $S H$-wave velocities in terms of incidence angle, $\varphi$, for synthetic materials, which have been shown in Table 1. The velocities of body waves in an isotropic material (Mat \#1) are independent of the direction of propagation, while it is different for the other materials. This behavior is expected and can be seen in these figures. A magnitude of $\nu_{P 1}$ at an incident angle of $\pi / 2$ is $\sqrt{A_{1111} / \rho}$, while it is equal to $\sqrt{A_{3333} / \rho}$ for incident angle of zero. The maximum compressional body wave velocity of the first kind belongs to Mat \#4 (see Figure 9a). As illustrated in Figure $9 \mathrm{~b}$, the velocity difference of $\nu_{P 2}$ for Mat \#2 and Mat \#5 is negligible, which is indicated in Table 1 , too. Furthermore, the maximum value of $\nu_{S V}$ occurs in the incident angle equal to $\varphi=\pi / 4$ and its related odd coefficients. In addition, according to Eqs. (29) and $(31), v_{S V}=\sqrt{C_{1313} / \rho}$ in the isotropic plane and the plane parallel to the material axis of symmetry, as seen in Figure 9c. It has been shown in Figure 9d that the $S H$-wave velocity of Mat \#1 and Mat \#5 is the

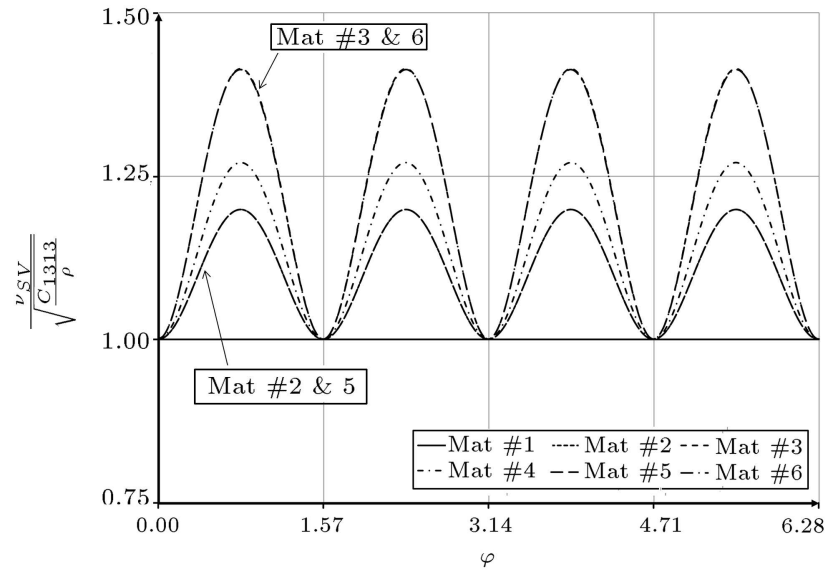

Figure 9c. Normalized $S V$-wave velocity versus incidence angle, $n=0.5$, and $\omega=50 \mathrm{rad} / \mathrm{sec}$.

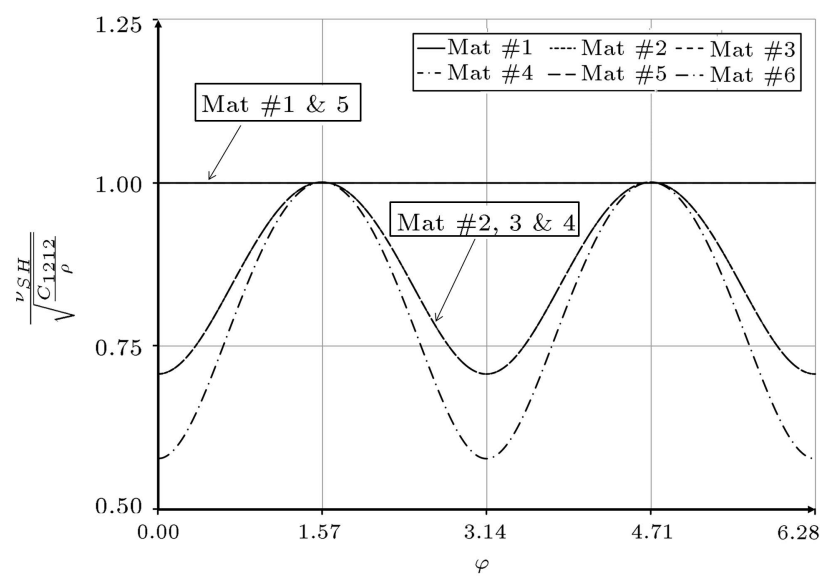

Figure 9d. Normalized $S H$-wave velocity versus incidence angle, $n=0.5$.

same. Also, this is true of Mat \#2 and Mat \#4. This result is related to the values of material coefficients $C_{1212}$ and $C_{1313}$. Figure 10 illustrates the $P 1-, P 2-$, and $S V$-wave attenuation coefficients versus incidence angle $\varphi$. The results demonstrate that the responses are strongly influenced by both the degree of anisotropy of the material and the incident angle. The maximum and minimum values of the attenuation coefficient of $P 1$-wave occur at incident angles equal to $\pi / 2$ and 0 , respectively. However, a reverse rule occurs for $P 2-$ wave. Besides, according to Eqs. (29), (31), and (33), the attenuation coefficient of $S V$-wave is expected to be zero when the wave propagates in the vertical and horizontal direction as well as in the saturated isotropic material. As shown in Figure 10c the numerical results also confirm this. Furthermore, it can be observed from this figure that Mat \#2 and Mat \#5 have the same attenuation coefficient, as their velocity. Another point is that in Mat \#3, the attenuation coefficient of $S V$-wave, besides at $\varphi=0$ and $\varphi=\pi / 2$ vanishes at $\varphi=\pi / 4$.

Figure 11 finally depicts the frequency depen- 


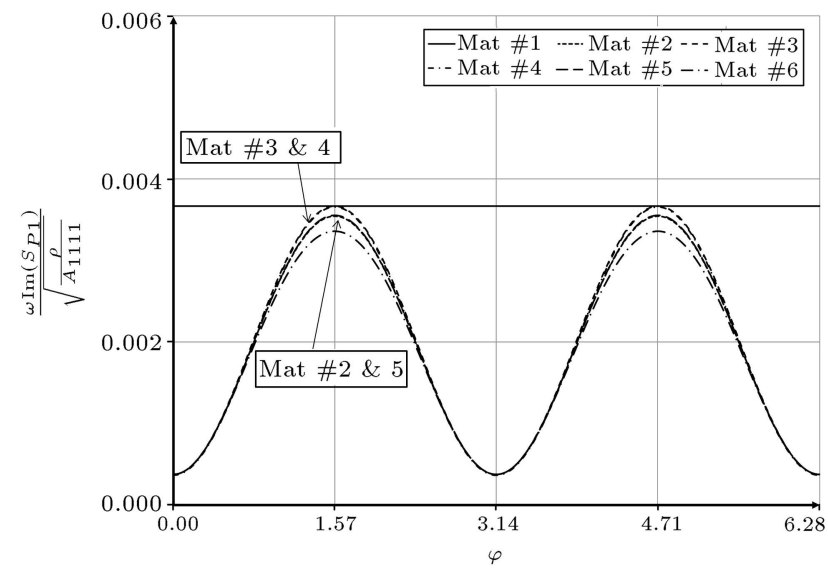

Figure 10a. Normalized $P 1$-wave attenuation coefficient versus incidence angle, $n=0.5$ and $\omega=50 \mathrm{rad} / \mathrm{sec}$.

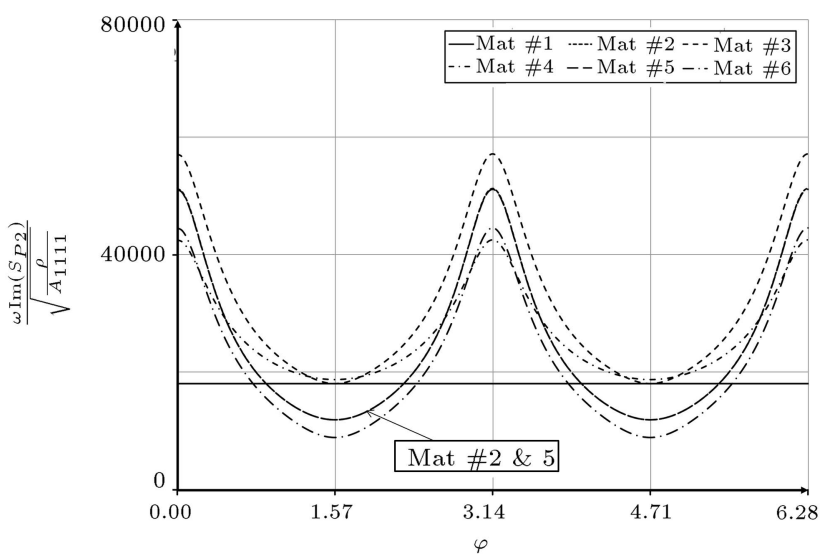

Figure 10b. Normalized $P 2$-wave attenuation coefficient versus incidence angle, $n=0.5$ and $\omega=50 \mathrm{rad} / \mathrm{sec}$.

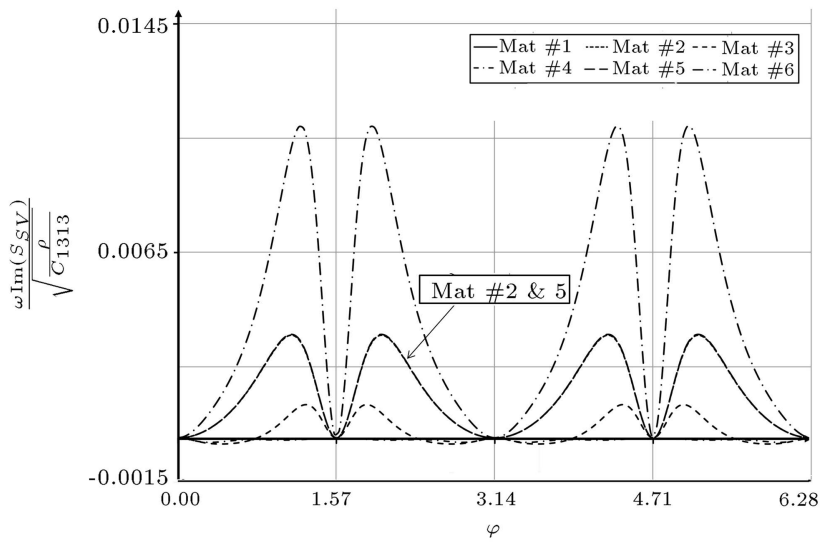

Figure 10c. Normalized $S V$-wave attenuation coefficient versus incidence angle, $n=0.5$ and $\omega=50 \mathrm{rad} / \mathrm{sec}$.

dence of the velocities and related attenuation coefficients for different values of $k_{1} / k_{3}$ (with $k_{1}=3.55 \times$ $10^{-12} \mathrm{~m}^{2}$ ) when the waves travel in a vertical direction. As can be seen, while both velocity and attenuation coefficient of $P 2$-wave are significantly affected by $k_{1} / k_{3}$, only the imaginary part of $S_{P 1}$ are mostly

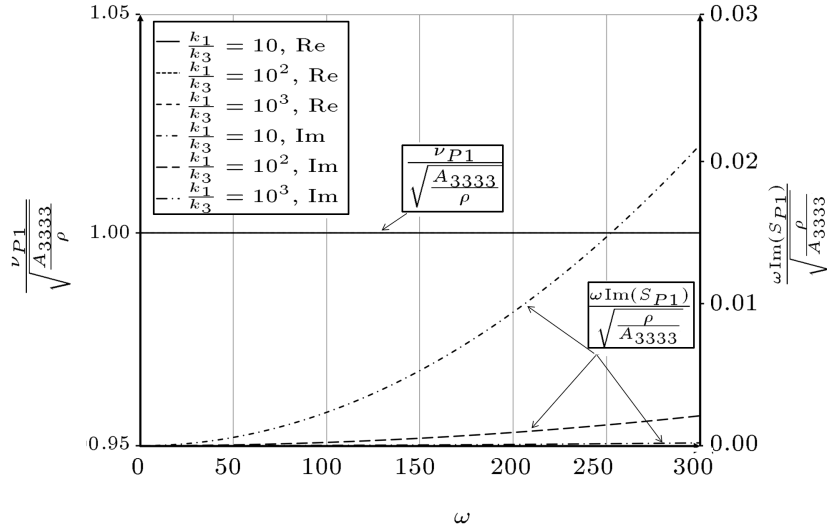

Figure 11a. Normalized $P 1$-wave velocity and corresponding attenuation coefficient versus frequency $(\mathrm{rad} / \mathrm{sec})$ for the different ratio of $k_{1} / k_{3}$, Mat $\# 4, n=0.2$, and $\varphi=0$.

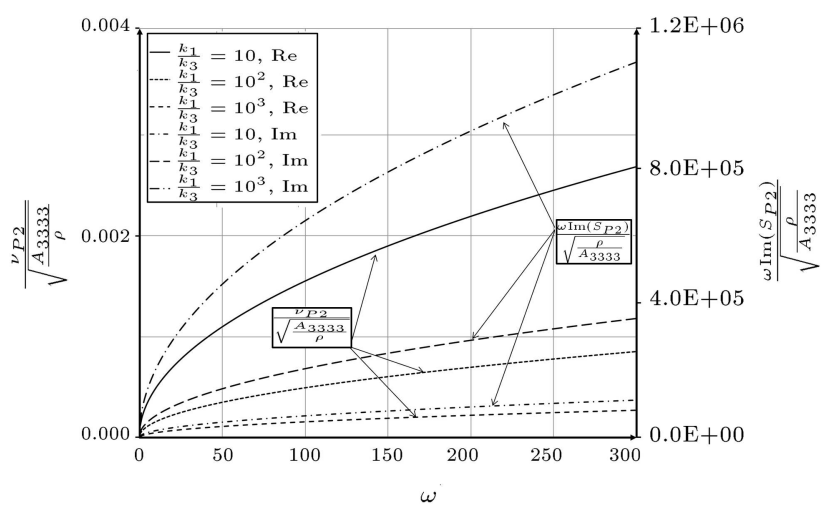

Figure 11b. Normalized $P 2$-wave velocity and corresponding attenuation coefficient versus frequency $(\mathrm{rad} / \mathrm{sec})$ for the different ratio of $k_{1} / k_{3}$, Mat \#4, $n=0.2$, and $\varphi=0$.

affected by $k_{1} / k_{3}$ and it has a minor effect on the velocity of the fast compressional wave. Moreover, the decrease in the permeability along the $x_{3}$-axis resulted in the decrease of the $P 2$-wave velocity, and increase of the corresponding attenuation coefficient (see Figure 11b).

Furthermore, we have carried out separate numerical studies, the result of which reveal that the effect of the value of $k_{3}$ and consequently changing $k_{1} / k_{3}$ on velocity and corresponding attenuation coefficient of $P 1$ - and $P 2$-waves is negligible in the horizontal plane. Besides, since $S_{P 1}$ and $S_{P 2}$ in isotropic plane depend on $k_{1}$, Eq. (31), we expected that changes in $k_{1} / k_{3}$ would not affect the velocities and corresponding attenuation coefficients of these waves. In addition, by referring to the equations presented in the previous section, it may be concluded that the velocity of $S V$-wave in the horizontal and vertical plane is neither a function of frequency nor a function of permeability. Therefore, in accordance to these findings, the relevant figures are not plotted. 


\section{Conclusion}

The propagation of plane harmonic body waves in transversely isotropic linear poroelastic fluid-saturated materials with a depth-wise axis of material symmetry for both fluid and solid has been investigated in this study. Therefore, based on Biot's theory, the potential function method in the framework of simplified $\mathbf{u}$ $p$ formulation has been used. The longitudinal and transverse velocities and corresponding attenuation coefficients have been extracted from the presented body wave equations. To demonstrate the validity of the analytical solution developed in this study, degeneration to zero porosity has been presented to provide interesting comparisons with the solutions given in the literature. Moreover, the expression of velocity and attenuation coefficient of body waves in the isotropic plane and the plane perpendicular to it, have been derived in explicit form. Also, the simplified form of the relations has been obtained for saturated isotropic materials and incompressible constituents. Furthermore, the effects of hydraulic and mechanical parameters of materials on the velocity of propagation and related attenuation coefficient of the waves have been investigated in more detail. To do so, a variety of synthetic poroelastic transversely isotropic materials have been provided, and the dependency of wave motion to these parameters is illustrated by plotting the graphs.

The figures provided for different frequencies give a comprehensive picture of the effect of these parameter on the velocities and, therefore, on the corresponding attenuations. It has been found that the first and the second kind of dilatational as well as vertical shear waves in transversely isotropic saturated media, in general, are dispersive and attenuated. The behaviour of the wave of the second kind is similar to that of heat conduction phenomenon, therefore the attenuation is very high. The attenuation coefficient of $S V$-wave for some special cases tends to zero. Also, the excellent agreement between the analytical equations obtained for the special cases and the numerical results has been illustrated. In addition, the effects of porosity and permeability have been demonstrated. Moreover, the result of the separate numerical studies show the effects of the degree of material anisotropy and wave incident angle on body wave velocities and related attenuation coefficients. The analytical and numerical results represent that the influence of anisotropy characteristics should be considered for wave propagation in poroelastic transversely isotropic materials.

\section{Nomenclature}

$\begin{array}{ll}A_{i j k l} & \text { Material constants } \\ C_{i j k l} & \text { Elasticity constants }\end{array}$

$d$

E

$E^{\prime}$

F

G

$G^{\prime}$

$i=\sqrt{-1}$

$K_{f}$

$K_{s}$

$k_{1}$

$k_{3}$

$M$

$m_{i}(i=1,2,3)$ Wave normal component

$n \quad$ Porosity

$p \quad$ Pore fluid pressure

$q \quad$ Specific flux

$S \quad$ Slowness

$t \quad$ Time variable

$U_{i}(i=1,2,3)$ Displacement component of fluid phase

$u_{i}(i=1,2,3) \quad$ Displacement component of solid phase

$V$

$V_{f} \quad$ Volume of the interconnected pores

$V_{s} \quad$ Volume of the solid phase

$w \quad$ Relative fluid to solid displacement

$\alpha_{1} \quad$ Biot's effective stress coefficient in the horizontal plane

$\alpha_{3} \quad$ Biot's effective stress coefficient in the vertical plane

$\delta_{i j} \quad$ Kronecker delta

$\varepsilon_{i j}(i, j=1,2,3)$ Strain tensor

$\zeta \quad$ Variation of fluid content

$\eta \quad$ Dynamic viscosity of the fluid

$\lambda \quad$ Lame's constant

$\mu \quad$ Lame's constant

$\rho \quad$ Mass density of the mixture

$\rho_{f} \quad$ Mass density of the fluid

$\rho_{s} \quad$ Mass density of the matrix

$\sigma_{i j}(i, j=1,2,3)$ Stress tensor

$\sigma_{i j}^{s}(i, j=1,2,3)$ Stress tensor of matrix

$\nu$

Poisson's ratio characterizing the lateral strain response in the plane of transverse isotropy to stress acting parallel to it 


$\begin{array}{ll}\nu^{\prime} & \text { Poisson's ratio characterizing the } \\ & \text { lateral strain response in the plane of } \\ & \text { transverse isotropy to stress acting } \\ & \text { normal to it } \\ v & \text { Kinematic viscosity of the fluid } \\ \chi & \text { Scalar potential function } \\ \omega & \text { Angular frequency }\end{array}$

\section{References}

1. Selvadurai, A.P.S., Mechanics of Poroelastic Media, Springer Science+Business Media, Dordrecht (1996).

2. Degrande, G., De Roeck, G., Van Den Broeck, P., and Smeulders, D. "Wave propagation in layered dry, saturated and unsaturated poroelastic media", International Journal of Solids Structures, 35, pp. 47534778 (1998).

3. Hamidzadeh, H.R., Dai, L., and Jazar, R.N., Wave Propagation in Solid and Porous Half-space Media, Springer Science+Business Media, New York (2014).

4. Schanz, M. "Poroelastodynamics: Linear models, analytical solutions, and numerical methods", Applied Mechanics Reviews ASME, 62, pp. 030803-1-03080315 (2009).

5. Biot, M.A. "General theory of three-dimensional consolidation", Journal of Applied Physics, 12, pp. 155164 (1941).

6. Biot, M.A. "Theory of elasticity and consolidation for a porous anisotropic solid", Journal of Applied Physics, 26, pp. 182-185 (1955).

7. Biot, M.A. "Theory of deformation of a porous viscoelastic anisotropic solid", Journal of Applied Physics, 27(5), pp. 459-467 (1956a).

8. Biot, M.A. "Theory of propagation of elastic waves in a fluid-saturated porous solid I. Low-frequency range", Journal of the Acoustical Society of America, 28(2), pp. 168-178 (1956b).

9. Biot, M.A. "Theory of propagation of elastic waves in a fluid-saturated porous solid II. higher frequency range", Journal of the Acoustical Society of America, 28(2), pp. 179-191 (1956c).

10. De Boer, R. "Development of porous media theoriesA brief historical review", Transport in Porous Media, 9, pp. 155-164 (1992).

11. Plona, T.J. "Observation of a second bulk compressional wave in porous medium at ultrasonic frequencies", Applied Physics, 36(4), pp. 259-261 (1980).

12. Van Dalen, K.N., Multi-Component Acoustic Characterization of Porous Media, Uitgeverij BOX Press, Oisterwijk, The Netherlands (2011).

13. Bonnet, G. "Basic singular solutions for a poroelastic medium in the dynamic range", Journal of the Acoustical Society of America, 82(5), pp. 1758-1762 (1987).

14. Zienkiewicz, O.C. and Shiomi, T. "Dynamic behaviour of saturated porous media: The generalized Biot formulation and its numerical solution", International Journal for Numerical and Analytical Methods in Geomechanics, 8, pp. 71-96 (1984).

15. Zienkiewicz, O.C., Chang, C.T., and Bettess, P. "Drained, undrained, consolidating and dynamic behaviour assumptions in soils", Geophysics, 30(4), pp. 385-395 (1980).

16. Schanz, M. and Struckmeier, V. "Wave propagation in a simplified modeled poroelastic continuum: Fundamental solutions and a time domain boundary element formulation", International Journal for $\mathrm{Nu}$ merical Methods in Engineering, 64, pp. 1816-1839 (2005).

17. Sahebkar, K. and Eskandari-Ghadi, M. "Timeharmonic response of saturated porous transversely isotropic half-space under surface traction", Journal of Hydrology, 537, pp. 61-73 (2016a).

18. Xu, C., Song, J., Du, X., and Zhang, Z. "A completely explicit finite element method for solving dynamic $u-p$ equations of fluid-saturated porous media", Soil Dynamics and Earthquake Engineering, 97, pp. 364376 (2017).

19. Geertsma, J. and Smit, D. "Some aspects of elastic wave propagation in a fluid-saturated porous solid", Geophysics, 27, pp. 169-181 (1961).

20. Deresiewicz, H. "The effect of boundaries on wave propagation in a liquid-filled porous solid: I. Reflection of plane waves at a free plane boundary (nondissipative case)", Bulletin of the Seismological Society of America, 50, pp. 599-607 (1960).

21. Deresiewicz, H. "The effect of boundaries on wave propagation in a liquid-filled porous solid: IV. Surface waves in a half-space", Bulletin of the Seismological Society of America, 52, pp. 627-638 (1962).

22. Deresiewicz, H. and Rice, J.T. "The effect of boundaries on wave propagation in a liquid-filled porous solid: III. Reflection of plane waves at free plane boundary (general case)", Bulletin of the Seismological Society of America, 52, pp. 595-625 (1962).

23. Beskos, D.E., Vgenopoulou, I., and Providakis, C.P. "Dynamics of saturated rocks. II: Body waves", Journal of Engineering Mechanics, 115, pp. 996-1016 (1989a).

24. Beskos, D.E., Providakis, C.P., and Woo, H.S. "Dynamics of saturated rocks. III: Rayleigh waves", Journal of Engineering Mechanics, 115, pp. 1017-1034 (1989b).

25. Amadei, B. "Importance of anisotropy when estimating and measuring in situ stresses in rock", International Journal of Rock Mechanics and Mining Science \& Geomechanics Abstracts, 33(3), pp. 293-325 (1996).

26. Schmitt, P.D. "Acoustic multipole logging in transversely isotropic poroelastic formations", Journal of the Acoustical Society of America, 86, pp. 2397-2421 (1989). 
27. Sharma, M.D. and Gogna, M.L. "Wave propagation in anisotropic liquid-saturated porous solid", Journal of the Acoustical Society of America, 90, pp. 1068-1073 (1991).

28. Carcione, J.M. "Wave field in real media: wave propagation in anisotropic, anelastic and porous media", Handbook of Geophysical Exploration, Seismic Exploration, 3rd Ed., Elsevier Science Ltd (2015).

29. Carcione J.M. "Wave propagation in anisotropic saturated porous media: Plane-wave theory and numerical simulation", Journal of the Acoustical Society of America, 99, pp. 2655-2666 (1996).

30. Ba, Z., Kang, Z., and Lee, V.W. "Plane strain dynamic responses of a multi-layered transversely isotropic saturated half-space", International Journal of Engineering Science, 119, pp. 55-77 (2017).

31. Zhang, Z. and Pan, E. "Time-harmonic response of transversely isotropic and layered poroelastic halfspaces under general buried loads", Applied Mathematical Modelling, 80, pp. 426-453 (2020).

32. Achenbach, J.D., Wave Propagation in Elastic Solids, North Holland Publishing Company, Amsterdam, The Netherlands (1973).

33. Lu, J.F. and Jeng, D.S. "A half-space saturated poroelastic medium subjected to a moving point load", International Journal of Solids and Structures, 44(2), pp. 573-586 (2007).

34. Pooladi, A., Rahimian, M., and Pak, R.Y.S. "Threedimensional time-harmonic fundamental solutions for a fluid-saturated poroelastic half-space with partially permeable free surface", International Journal for Numerical and Analytical Methods in Geomechanics, 40, pp. 1903-1935 (2016).

35. Lekhnitskii, S.G., Theory of Elasticity of an Anisotropic Body, Mir Publishers, Moscow (1981).

36. Eskandari-Ghadi, M. "A complete solution of the wave equations for transversely isotropic media", Journal of Elasticity, 81, pp. 1-19 (2005).

37. Raoofian-Naeeni, M. and Eskandari-Ghadi M. "A potential method for body and surface wave propagation in transversely isotropic half- and full-spaces", Civil Engineering Infrastructures Journal, 49(2), pp. 263288 (2016).

38. Pooladi, A., Rahimian, M., and Pak, R.Y.S. "Poroelastodynamic potential method for transversely isotropic fluid-saturated poroelastic media", Applied Mathematical Modeling, 50, pp. 177-199 (2017).

39. Sahebkar, K. and Eskandari-Ghadi, M. "Displacement ring load Green's functions for saturated porous transversely isotropic tri-material full-space", International Journal for Numerical and Analytical Methods in Geomechanics, 41(3), pp. 359-381 (2016b).

40. Sahebkar, K. and Eskandari-Ghadi, M. "Dynamic behaviour of an infinite saturated transversely isotropic porous media under fluid-phase excitation", Soil Dynamics and Earthquake Engineering, 107, pp. 390-406 (2018).
41. Jafarzadeh, A. and Eskandari-Ghadi, M. "Surface load dynamic solution of saturated transversely isotropic multilayer half-space", International Journal for $\mathrm{Nu}$ merical and Analytical Methods in Geomechanics, 43(9), pp. 1655-1685 (2019).

42. Ba, Z., Mengtao, W., and Liang, J. "3D dynamic responses of a multi-layered transversely isotropic saturated half-space under concentrated forces and pore pressure", Applied Mathematical Modelling, 80, pp. 859-878 (2020).

43. Liang, J., Mengtao, W., and Ba, Z. "Threedimensional dynamic Green's functions for transversely isotropic saturated half-space subjected to buried loads", Engineering Analysis with Boundary Elements, 108, pp. 301-320 (2019).

44. Biot, M.A. "Mechanics of deformation and acoustic propagation in porous media", Journal of Applied Physics, 33, pp. 1482-1498 (1962).

45. Biot, M.A. and Willis, D.G. "The elastic coefficients of the theory of consolidation", Journal of Applied Mechanics, 24, pp. 594-601 (1957).

46. Sneddon, I.N., Elements of Partial Differential Equations (International series in pure \& applied mathematics), McGraw-Hill Inc., International Edition, US (1964).

47. Wang, C.D., Wang, W.J., Lin, Y.T., and Ruan, Z.W. "Wave propagation in an inhomogeneous transversely isotropic material obeying the generalized power law model", Archive of Applied Mechanics, 82, pp. 919936 (2011).

48. Chadwick, P. and Seet, L.T.C. "Wave propagation in a transversely isotropic heat-conducting elastic material", Mathematika, 17, pp. 255-274 (1970).

49. Wang, C.D., Lin, Y.T., Jeng, Y.S., and Ruan, Z.W. "Wave propagation in an inhomogeneous crossanisotropic medium", International Journal for $\mathrm{Nu}$ merical and Analytical Methods in Geomechanics, 34, pp. 711-732 (2010).

50. Schanz, M. and Pryl, D. "Dynamic fundamental solutions for compressible and incompressible modeled poroelastic continua", International Journal of Solids and Structures, 41, pp. 4047-4073 (2004).

51. Cheng, A.H.-D., Poroelasticity, Springer International Publishing, Switzerland (2016).

52. Schanz, M. and Cheng, A.H.-D. "Transient wave propagation in a one-dimensional poroelastic column", Acta Mechanica, 145, pp. 1-18 (2000).

\section{Biographies}

Mohammadreza Mahmoodian received his MSc degree in Structural Engineering from the University of Science and Culture, Tehran, Iran in 2010. He is admitted as a talented $\mathrm{PhD}$ candidate in Structural Engineering at the University of Science and Culture. His research interests include the theory of elasticity, 
wave propagation in solids/fluids, and mechanics of porous media. He is now working on wave propagation in transversely isotropic poroelastic fluid-saturated media. He has published three papers in scientific journals and several conference papers so far.

Morteza Eskandari-Ghadi holds two PhDs one of which is in Structural Engineering from the University of Tehran, Iran, and the other is in the Mechanics of Structures from the University of Colorado at Boulder, USA. Although he has undertaken some research related to composite materials, he is a specialist in wave propagation in solids, especially in transversely isotropic materials. His original researches were the source of about 100 articles which were published in international journals. Some of these studies are considered as benchmarks for other researches in the related areas. His set of potential functions for solving the system of equations of motion in transversely isotropic materials published in the Journal of Elasticity is considered as to be his most outstanding work. He is the author/co-author of six books in the field of continuum mechanics, the theory of elasticity, and multivariable functions. He is now a Professor in the School of Civil Engineering at the University of Tehran, Iran.

Ali Nikkhoo, currently an Associate Professor at the University of Science and Culture, Tehran, Iran, received his BSc in Civil Engineering from the Isfahan University of Technology, Isfahan, Iran, in 2000, and MSc and PhD degrees from Sharif University of Technology, Tehran, Iran, in 2002 and 2008, respectively. His research interests are smart materials and structures, structural dynamics, and computational mechanics. Near 40 contributions have been published in the international journals. 